\title{
Effect of the loading mode on the evolution of the deformation mechanisms in randomly textured magnesium polycrystals - comparison of experimental and modeling results
}

K. Máthis ${ }^{1, *}$, G. Csiszár ${ }^{2,3}$, J. Čapek ${ }^{1}$, J. Gubicza², B. Clausen ${ }^{4}$, P. Lukáš ${ }^{5}$, A. Vinogradov ${ }^{6}$, S. R. Agnew $^{7}$

${ }^{1}$ Department of Physics of Materials, Faculty of Mathematics and Physics, Charles University, Ke Karlovu 5, 12116 Prague, Czech Republic

${ }^{2}$ Department of Materials Physics, Faculty of Science, Eötvös Loránd University, Pázmány P. sétány 1/A, H-1117 Budapest, Hungary

${ }^{3}$ Department of Phase Transformations, Thermodynamics and Kinetics, Max Planck Institute for Intelligent Systems, Heisenbergerstr. 3, 70569 Stuttgart, Germany

${ }^{4}$ Los Alamos National Laboratory, Lujan Neutron Scattering Center, Los Alamos, NM 87545

USA

${ }^{5}$ Nuclear Physics Institute of the Academy of Sciences of the Czech Republic, 25068 Řež, Czech Republic

${ }^{6}$ Laboratory of Physics of Strength of Materials and Intelligent Diagnostic Systems, Togliatti State University, 445667 Togliatti, Russia

${ }^{7}$ School of Engineering and Applied Science, University of Virginia, 116 Engineer's Way, Charlottesville, VA 22904, USA

\begin{abstract}
A detailed analysis of the loading mode dependence of the deformation mechanisms in randomly textured cast magnesium is presented. An elasto-plastic self-consistent model (EPSC) is used to model the dislocation slip and twinning activity, respectively. The results are quantitatively compared with experimental data obtained by in-situ neutron diffraction (ND) and acoustic emission (AE). Both EPSC calculations and ND line profile analysis show an increased activity of prismatic slip with increasing strain and a loading mode dependence of the activation of the second-order pyramidal slip. The AE measurements and the modeling indicate a difference in the number of nucleated twin variants and the twinned volume in tension and compression.
\end{abstract}

Keywords: twinning; polycrystalline material; crystal plasticity; nondestructive evaluation;

\footnotetext{
* Corresponding author. E-mail: mathis@ met.mff.cuni.cz; Phone: +420-221-911-458; Fax: +420-221-911-490
} 


\section{Introduction}

Magnesium alloys represent the lowest density structural metals (along with toxic beryllium) and, thus, is a highly interesting material for transportation industry, where the fuel saving achieved through weight reduction is among the most important tasks (Mordike and Ebert, 2001; Hirsch and Al-Samman, 2013). Nevertheless, their widespread application is limited by the higher production costs in comparison to aluminium alloys and steels, caused especially by low formability of magnesium alloys at ambient temperature (Hirsch and Al-Samman, 2013). Despite the efforts of the scientific community in the last several decades the study of the deformation properties of magnesium alloys is still a challenging task. Their hexagonal closed packed (hcp) structure and ratio of the crystallographic axes $c / a$ close to ideal cause a unique deformation behavior completely different from the other cubic (e.g. fcc, bcc) metals. At room temperature, the $(0001)\langle 11 \overline{2} 0\rangle$ basal slip is the most easily activated system, followed by $\{10 \overline{1} 0\}\langle 11 \overline{2} 0\rangle$ prismatic and first-order $\{10 \overline{1} 1\}\langle 11 \overline{2} 0\rangle$ pyramidal slip systems (Avedesian and Baker, 1999; Chapuis and Driver, 2011). Since all of these systems combined provide deformation within the basal plane and offer not more than four independent slip systems, the von Mises criterion of five independent slip systems for homogeneous deformation is not fulfilled and a deformation along the $c$-axis is not possible. The slip in the second-order $(\{11 \overline{2} 2\}\langle\overline{1} \overline{1} 23\rangle)$ pyramidal system would satisfy the von Mises criterion. Nevertheless, the activation of the $\langle c+a\rangle$ dislocations is rather difficult owing to the high critical resolved shear stress (CRSS) (Chapuis and Driver, 2011). Thus, deformation twinning is a preferred mechanism that accommodates deformation out of the basal plane. In magnesium, the $\{10 \overline{1} 2\}$-type extension twinning, associated with extension along the $c$-axis and reorientation of the lattice by $86.3^{\circ}$ and $\{10 \overline{1} 1\}$-type compression twinning, resulting in contraction along the $c$-axis and tilting of the lattice by $56^{\circ}$ are the most often reported and studied twinning mechanisms (Christian and Mahajan, 1995).

The activation of the above listed mechanisms significantly depends on the various microstructural features (initial texture (Kelley and Hosford, 1968), grain size (Muránsky et al., 2010a; Dobroň et al., 2011), alloying content (Akhtar and Teghtsoonian, 1969; Cáceres and Lukáč, 2008; Stanford and Barnett, 2013)) and the experimental conditions (strain path (Cáceres et al., 2008; Proust et al., 2009), strain rate and testing temperature (Máthis et al., 2006; Khan et 
al., 2011; Ghaderi et al., 2013)). The dependence of the twinning on the initial texture manifests macroscopically in the well-documented plastic anisotropy (different strength in different directions) and asymmetry (different yield strength in tension and compression along a particular direction). On the microscopic scale, the orientation of the surrounding grains significantly influences the nucleated twin variant and its growth. As it was proved recently both experimentally (Mu et al., 2012) and theoretically (Barnett et al., 2013), that the twin variant which nucleates with the highest probability is the one which requires the least accommodation work in the neighboring grain and for which the accommodation of the twinning strain is the easiest. For example, the growth of the twin variants which induce hard deformation modes, such as prismatic slip, in the adjacent grains, is hindered.

The study of the dislocation slip is also essential. There is a general agreement in the key role of the basal $\langle a\rangle$ slip in the plasticity of magnesium. Nevertheless, numerous papers highlight the onset of the prismatic $\langle a\rangle$ slip in the vicinity of the macroscopic yield point (e.g. (Agnew et al., 2006; Clausen et al., 2008; Balik et al., 2012) and its role in the twin growth (Xu et al., 2013). Despite the high resolved shear stress of the second-order pyramidal slip system at ambient temperatures, the $\langle c+a\rangle$ slip was found active during later stage of the compressive deformation, when the primary twinning mechanism is exhausted (El Kadiri and Oppedal, 2010; Oppedal et al., 2012a). Naturally, the interaction between twins and dislocations should also be taken into account. First of all, the twin boundaries can act as barriers for gliding dislocations (Serra and Bacon, 2010). Furthermore, the matrix dislocations can transmute upon the passage of a twin front and cause latent hardening in the twin, as it was suggested recently by Niewczas and El Kadiri \& Oppedal (Niewczas, 2007; El Kadiri and Oppedal, 2010; Oppedal et al., 2012a).

In the last decade, several different theoretical models were worked out for description of twinning and dislocation slip phenomena in hexagonal materials. The atomistic calculations (Serra et al., 1991; Tomé et al., 2011; Xu et al., 2013) describes the fundamental mechanisms of twin nucleation, growth and twin boundary - dislocation interactions. The various crystalplasticity models use different approaches, as Taylor model (e.g. (Levesque et al., 2010)), finite element method (Staroselsky and Anand, 2003; Fernandez et al., 2011; Hama and Takuda, 2011; Mayama et al., 2011) or self-consistent models (Lebensohn and Tome, 1993; Agnew et al., 2006; Clausen et al., 2008; Proust et al., 2009; Oppedal et al., 2012a; Wang et al., 2013a; Wang et al., 2013b). The use of the self-consistent models has developed particularly. Early examples focused 
only on texture evolution ((Agnew et al., 2001; Styczynski et al., 2004), then additional focus was placed on anisotropy, strain hardening, and the twinning caused reorientation during monotonic loading (Lebensohn and Tome, 1993; Agnew and Duygulu, 2005; Clausen et al., 2008; Neil et al., 2010). The most recent models can also successfully describe the stress-strain behavior during strain-path change (Proust et al., 2009), including the de-twinning phenomenon (Wang et al., 2013b), and the twin - dislocation interaction and the dislocation transmutation (Oppedal et al., 2012a).

The experimental study of the deformation mechanisms includes both $e x$-situ (e.g. optical light, scanning or transmission electron microscopy) and in-situ techniques (e.g. diffraction methods, acoustic emission etc.). The main drawback of microscopy methods in studying twinning and dislocations is the relatively small observed volume in the specimen. On contrary, the irradiated volume in the diffraction experiments provides statistically representative data. The X-ray line profile analysis, pioneered by Ungár et al. (Ungár, 2004), was successfully used for ex-situ analysis of the temperature dependence of the dislocation structure evolution during uniaxial tensile test of magnesium alloys (Máthis et al., 2006; Gubicza et al., 2010). The neutron diffraction (ND) technique was used first by Gharghouri et al. for study of twinning in Mg-Al alloy (Gharghouri et al., 1999). In this type of experiment, the overall twinned volume can be determined from the intensity variations of particular peaks, caused by the crystal lattice reorientation during twinning (Gharghouri et al., 1999; Muránsky et al., 2010b; Agnew et al., 2013). Furthermore, the activity of a particular slip system manifests as a deviation of the lattice strains from the ideally elastic response (Agnew et al., 2006). Muránsky (Muránsky et al., 2010a) introduced acoustic emission (AE) as a useful complementary experimental technique to ND during in-situ testing of the wrought Mg alloy, ZM20. The main advantages of the method are the high time resolution and sensitivity to twin nucleation and collective dislocation motion (Lou et al., 2007). A recent statistical method worked out by Pomponi \& Vinogradov (Pomponi and Vinogradov, 2013) was successfully applied for the determination of the dominant deformation mechanisms from the AE signal at the various stages of deformation (Vinogradov et al., 2013a; Vinogradov et al., 2013b).

The experimental validation of the constitutive modeling results includes comparison of calculated and experimental stress-strain curves and diffraction data (pole figures, lattice strain evolution) (Agnew et al., 2006; Muránsky et al., 2010b). 


\section{Experimental procedures and modeling}

\subsection{Tensile and compression testing}

Randomly textured cast polycrystalline magnesium was used for the experiments. The inverse pole figures (IPF), measured using neutron diffraction and characterizing the initial state of the specimens (Fig. 1), do not show any preferential orientation. The moderate grain size of $110 \pm 5$ $\mu \mathrm{m}$ was achieved with adding $1 \mathrm{wt} . \% \mathrm{Zr}$ to the melt. The testing was carried out using cylindrical specimens with a diameter of $9 \mathrm{~mm}$ and gauge length of $20 \mathrm{~mm}$. Tensile and compression testing were carried out using a horizontal $250 \mathrm{kN}$ capacity load frame at a strain rate of $1 \mathrm{x}^{-3} 0^{-3} \mathrm{~s}^{-1}$ in strain control mode. In order to collect ND data with good enough statistics, the tests were stopped at predefined strain levels $(0.1,0.5,1,2,3,4,5,6 \%)$ for approx. 60 min.

\subsection{Neutron diffraction experiments}

In-situ neutron diffraction measurements were carried out at the SMARTS engineering instrument (Bourke et al., 2002) in the Lujan Neutron Scattering Center. The mutual orientation of the longitudinal axis of the sample and the incident beam was $45^{\circ}$. Two detector banks were 
positioned at $\pm 90^{\circ}$ to the incident beam in order to record diffraction pattern in both parallel (bank 2) and perpendicular (bank 1) to the loading direction (for scheme of the experimental setup see (Clausen et al., 2008)). The first and the second detector banks are also referred to as radial and axial detectors, respectively, because of the measured component of the lattice strain.

The neutron diffraction patterns obtained for the $\mathrm{Mg}$ samples deformed up to the strain of $6 \%$ were evaluated by the Convolutional Multiple Whole Profile (CMWP) fitting method (Ribárik et al., 2004; Balogh et al., 2006). In this procedure, each diffraction pattern is fitted by the sum of a background spline and the convolution of the instrumental pattern and the theoretical line profiles related to crystallite (coherent scattering domain) size and dislocations (as manifested by the microstrain fields which surround them). The instrumental profiles were measured on a wellannealed CaF sample with sufficiently large grains. The theoretical profile functions used in this fitting procedure are calculated on the basis of a model of the microstructure, where the crystallites have spherical shape and log-normal size distribution and the microstrains are caused primarily by dislocations. It is noted that the fitting in the present study revealed that there is no considerable crystallite size broadening of the diffraction peaks due to the very large grain size of the investigated $\mathrm{Mg}$ samples (i.e. the cell size is larger than the detection limit of peak profile analysis). The twin boundary spacing also remained above the detection limit of the present peak profile analysis $(\sim 500 \mathrm{~nm})$. The CMWP fitting procedure gave the dislocation density $(\rho)$, and the parameters $q_{1}$ and $q_{2}$ which can be used for the determination of the fractions of dislocations in the different slip system families. In the following, a short description of the procedure used for the calculation of the dislocation fractions is given.

The strain broadening of the diffraction line profiles with the indices $h k l$ is determined by the mean-square-strain $\left(\left\langle\varepsilon_{\mathrm{g}, \mathrm{L}}^{2}\right\rangle\right)$ in the lattice in the direction of the diffraction vector $\boldsymbol{g}$. It is noted that in the present neutron diffraction study three indices will be used for the identification of reflections. If microstrains are caused by dislocations, the mean-square-strain can be given as:

$$
\left\langle\varepsilon_{g, L}^{2}\right\rangle \cong \frac{\rho C_{h k l} b^{2}}{4 \pi} f(\eta),
$$

where 


$$
\eta=\frac{1}{2} \exp \left(\frac{7}{4}\right) \frac{L}{R_{e}}
$$

and $\rho, b$ and $R_{\mathrm{e}}$ are the density, the modulus of Burgers vector and the effective outer cut-off radius of dislocations, respectively, $L$ is the Fourier variable and $C_{h k l}$ is the dislocation contrast (or orientation) factor (Wilkens, 1969). The function $f(\eta)$ is referred to as Wilkens function. In a crystal, the volume studied by X-ray diffraction contains numerous dislocations in different slip systems. Therefore, the mean-square-strain in eq. (1) should be averaged in the whole irradiated volume. For the simplicity, let us assume that the outer cut-off radius of dislocations, $R_{\mathrm{e}}$, is the same for all dislocations in the investigated volume. Then, the mean-square-strain can be given as:

$$
\left\langle\varepsilon_{g, L}^{2}\right\rangle \cong \frac{\rho\left\langle C_{h k l, j} b_{j}^{2}\right\rangle}{4 \pi} f(\eta),
$$

where $\rho$ is the average dislocation density in the irradiated volume and $\langle>$ indicates averaging over all the slip systems which are populated by dislocations with the weights equal to the contributions of the slip systems to the total dislocation density. The subscript $j$ denotes the $j$ th slip system in the crystal. In hexagonal crystals there are eleven families of slip systems on basal, prismatic and pyramidal planes with three different Burgers vectors (Klimanek \& Kuzel, 1988). The eleven dislocation slip system families can be classified into three groups based on their Burgers vectors: $b_{1}=\frac{1}{3}\langle\overline{2} 110\rangle(\langle a\rangle$ type $), b_{2}=\langle 0001\rangle(\langle c\rangle$ type $)$ and $b_{3}=\frac{1}{3}\langle\overline{2} 113\rangle(\langle c+a\rangle$ type $)$. Using the notation of Dragomir \& Ungár (Dragomir and Ungár, 2002), 4, 2 and 5 slip system families in the $\langle a\rangle,\langle c\rangle$ and $\langle c+a\rangle$ Burgers vector groups are considered (Table 1).

Table 1: The notation, the Burgers vector, the slip plane and the number of slip systems for each hexagonal slip system family (Dragomir and Ungár, 2002).

\begin{tabular}{|l|l|l|l|l|}
\hline Notation & $\begin{array}{l}\text { Slip plane and } \\
\text { edge/screw character }\end{array}$ & Burgers vector & Slip plane & $\begin{array}{l}\text { Number Burgers } \\
\text { of slip vector type } \\
\text { systems }\end{array}$ \\
\hline BE & Basal edge & $\frac{1}{3}\langle\overline{2110}\rangle$ & $\{0001\}$ & $3 \quad<$ <> \\
\hline
\end{tabular}




\begin{tabular}{|l|l|l|l|lc|} 
PrE & Prismatic edge & $\frac{1}{3}\langle 2110\rangle$ & $\{0110\}$ & 3 & $<\mathrm{a}>$ \\
\hline PyE & Pyramidal edge & $\frac{1}{3}\langle\overline{2} 110\rangle$ & $\{1011\}$ & 6 & $<\mathrm{a}>$ \\
\hline S1 & Screw & $\frac{1}{3}\langle\overline{2} 110\rangle$ & - & 3 & $<\mathrm{a}>$ \\
\hline Pr2E & Prismatic edge & $\langle 0001\rangle$ & $\{0110\}$ & 3 & $<\mathrm{c}>$ \\
\hline S3 & Screw & $\langle 0001\rangle$ & - & 1 & $<\mathrm{c}>$ \\
\hline Pr3E & Prismatic edge & $\frac{1}{3}\langle\overline{2} 113\rangle$ & $\{0110\}$ & 6 & $<\mathrm{c}+\mathrm{a}>$ \\
\hline Py2E & Pyramidal edge & $\frac{1}{3}\langle\overline{2} 113\rangle$ & $\{2112\}$ & 6 & $<\mathrm{c}+\mathrm{a}>$ \\
\hline Py3E & Pyramidal edge & $\frac{1}{3}\langle\overline{2} 113\rangle$ & $\{11 \overline{2} 1\}$ & 12 & $<\mathrm{c}+\mathrm{a}>$ \\
\hline Py4E & Pyramidal edge & $\frac{1}{3}\langle\overline{2} 113\rangle$ & $\{1011\}$ & 12 & $<\mathrm{c}+\mathrm{a}>$ \\
\hline S2 & Screw & $\frac{1}{3}\langle\overline{2} 113\rangle$ & - & 6 & $<\mathrm{c}+\mathrm{a}>$ \\
\hline
\end{tabular}

For randomly oriented polycrystalline materials it can be assumed that all slip systems in each family are populated equally with dislocations therefore $\left\langle C_{h k l, j} b_{j}^{2}\right\rangle$ can be expressed by the average dislocation contrast factors of the slip system families as:

$$
\left\langle C_{h k l, j} b_{j}^{2}\right\rangle=\sum_{j=1}^{11} f_{j} \bar{C}_{h k l, j} b_{j}^{2},
$$

where $\bar{C}_{h k l, j}$ is the average dislocation contrast factor for the jth slip system family which can be expressed as (Dragomir \& Ungár, 2002):

$$
\bar{C}_{h k l, j}=\bar{C}_{h k 0, j}\left(1+q_{1, j} z+q_{2, j} z^{2}\right),
$$

where $q_{1, \mathrm{j}}$ and $q_{2, \mathrm{j}}$ are two parameters depending on the anisotropic elastic constants of the crystal and the type of dislocation slip system family. $z=(2 / 3)(l / g a)^{2}$, where $a$ is the lattice constant in the basal plane. $\bar{C}_{h k 0, j}$ is the average dislocation contrast factor of the $h k 0$ type reflections for the 
$\bar{C}_{h k 0}^{m}\left(1+q_{1}^{m} z+q_{2}^{m} z^{2}\right) b_{m}^{2}=\sum_{j=1}^{11} f_{j} \bar{C}_{h k 0, j}\left(1+q_{1, j} z+q_{2, j} z^{2}\right) b_{j}^{2}$,

where $q_{1}^{m}$ and $q_{2}^{m}$ are the measured values of $q_{1}$ and $q_{2}$. The polynomials in the two sides of eq. (6) give the same values, if the coefficients of the terms with the same degrees are equal. This condition yields the following equations:

$$
\begin{aligned}
& q_{1}^{m}=\frac{\sum_{j=1}^{11} f_{j} \bar{C}_{h k 0, j} b_{j}^{2} q_{1, j}}{\sum_{j=1}^{11} f_{j} \bar{C}_{h k 0, j} b_{j}^{2}}, \\
& q_{2}^{m}=\frac{\sum_{j=1}^{11} f_{j} \bar{C}_{h k 0, j} b_{j}^{2} q_{2, j}}{\sum_{j=1}^{11} f_{j} \bar{C}_{h k 0, j} b_{j}^{2}}, \\
& \sum_{j=1}^{11} f_{j}=1 .
\end{aligned}
$$

There is no equation for $\bar{C}_{h k 0}^{m}$, since it is not an independent parameter in the evaluation of line profiles ( $\bar{C}_{h k 0}^{m}$ is multiplied with the dislocation density in eq. (1)). The eleven values of $f_{j}$ cannot be determined from the three formulas in eq. (7), therefore additional restrictions are made for $f_{j}$. It is assumed that in each Burgers vector group the non-zero fractions are equal. This assumption reduces the number of variables to three, which are denoted by $f_{a}, f_{c}$ and $f_{c+a}$. A computer 
program was elaborated in order to determine the distribution of dislocations among the different slip system families from $q_{1}^{m}$ and $q_{2}^{m}$ (Máthis et al., 2004). The program referred to as Hexburger first selects some slip system families from $\langle a\rangle$ dislocation group and for these slip systems the weights are $f_{a}$. For other slip systems in this group the weights are zero. This procedure is also carried out for $\langle c\rangle$ and $\langle c+a\rangle$ Burgers vector groups where the non-zero weights are $f_{c}$ and $f_{c+a}$, respectively. Inserting the theoretical values of $\bar{C}_{h k 0, j}, b_{j}, q_{1, j}$ and $q_{2, j}$ into eq. (7), the values of $f_{a}, f_{c}$ and $f_{c+a}$ are determined. If these fractions have positive values the program stores them as one of the possible solutions. The number of the possible selections from the dislocation slip systems equals $\left(2^{4}-1\right)\left(2^{2}-1\right)\left(2^{5}-1\right)=1395$. Finally, the positive solutions for the weights can be averaged for each slip system family, leading to the fractions of the eleven dislocation types. The fractions of the three Burgers vector groups, $h_{a}, h_{c}$ and $h_{c+a}$, are obtained by the summation of the fractions of the related slip system families.

\subsection{Acoustic emission measurements}

In a separate deformation test, a broadband $\mathrm{AE}$ sensor from Dakel company was mounted on the outside the gauge length using vacuum grease and an elastic band. The AE amplified by $40 \mathrm{~dB}$ in the frequency range $100-1200 \mathrm{kHz}$. The AE acquisition took place in a so-called data streaming regime, where the data were recorded continuously with 18 bits amplitude resolution and $2 \mathrm{MHz}$ sampling rate.

The classical AE testing includes a hit-based processing, when the 'useful' signal is extracted from the background using a threshold level and hit definition time (HDT) and reduced for waveforms, having characteristic parameters, as amplitude, duration, rise-time, counts etc. In materials science, this approach can be successfully applied for general characterization, when the main goal is the investigation of the influence of the experimental and material parameters on the deformation behavior (e.g. (Dobron̆ et al., 2007; Chmelik et al., 2007)). Nevertheless, the hitbased AE recording has several drawbacks: (i) in most cases, there is no direct link between the source mechanism and the AE parameters; (ii) the proper setting of the signal extracting parameters is rather difficult, when multiple, strong sources are simultaneously active. Since there is a general agreement that particular AE processes produce different waveforms (Scruby et al., 1981), the first issue is usually solved by use of a spectrum-based analysis, when the 
characteristic features of frequency domain of the waveforms are investigated. The threshold setting problem can be sorted out using continuous data streaming, when the raw signal is recorded without pre-defined threshold level and the characteristic parameters are evaluated during post-processing. The price for this solution is a huge amount of information arriving at 110 Msamples/s rate and the need for new approaches for quantitative characterization of the acquired time series. In the present work, the data were evaluated following the adaptive sequential $k$-means (ASK) procedure developed recently by Vinogradov et al. (Pomponi and Vinogradov, 2013; Vinogradov et al., 2013a; Vinogradov et al., 2013b). The continuously streamed data were sectioned into consecutive individual realizations ("frames") containing 2048 samples with 1024 overlapping points. Afterwards their power spectral density (PSD) function $G(f)$ was calculated for each frame. From the PSD function the AE energy per realization $E$ referred to $1 \Omega$ nominal impedance and median frequency $f_{m}$ was derived as follows: $E=\int_{f_{\min }}^{f_{\max }} G(f) d f$ and $\int_{0}^{f_{m}} G(f) d f=\int_{f_{m}}^{\infty} G(f) d f$. Furthermore, the normalized PSDs, defined as $\tilde{G}(f)=G(f) / E$ and obeying the condition $\int_{0}^{\infty} \tilde{G}(f) d f=1$ were calculated as input parameters for statistical analysis of the signals. Owing to the random character of the AE, there is a significant scatter in the waveforms and PSDs. Thus, the discrimination between signals stemming from different sources can only be done on the statistical basis. Various statistical procedures commonly referred to as "clustering" have been proposed in the literature (regardless of details, any clustering aims at grouping like objects and separating unlike ones based on a certain similarity measure), e.g. the $k$-means (Sibil et al., 2012), fuzzy $c$-means (Fotouhi et al., 2012), pattern recognition (Kontsos et al., 2011), hierarchical clustering (Lu et al., 2008) or neuron analysis ( $\mathrm{Lu}$ et al., 2008). The main advantages of the ASK clustering method applied in this work are the follows: (i) the number of clusters is data driven, i.e. it is not necessary to assume any number of active deformation processes in advance; (ii) the process is non-iterative, i.e. - AE signals are associated with a certain cluster sequentially based on their arrival time. We used the symmetric version of the Kullback-Liebler (KL) divergence $d_{K L}$ to distinguish between the normalized AE spectra $\tilde{G}_{i}$. (For further details see (Vinogradov et al., 2013b).) The analysis starts with the statistical characterization of the power spectrum density of the background noise, 
which gives the first reference point for comparison and the definition of the first cluster centroid. With the chosen distance $D\left(\tilde{G}_{i}, \tilde{G}_{j}\right)$ between all class members $\tilde{G}_{i}$, the mean distance $\bar{D}$ and the standard deviation $\sigma_{G G}$ is calculated to obtain the intra-cluster distance $R=\bar{D}+\alpha \sigma_{G G}$, where $\alpha \in(0 ; 3)$ is a constant set as 3 in our work (Vinogradov et al., 2013b). Every consecutive AE realization is then either assigned to the nearest cluster or used as a seed of a new cluster. It should be noted that the results of the cluster analysis should be verified by other experimental methods in order to warrant their right interpretation.

\subsection{Electron back-scattered diffraction analysis}

The specimens deformed to $1 \%$ of applied strain were polished by standard methods down to OPS colloidal silica suspension and finally electropolished in the Struers AC-2-II electrolyte for electron back-scattered diffraction (EBSD) investigations. The EBSD analysis was performed on a Quanta FEG microscope.

\subsection{EPSC modeling}

In the present work the EPSC deformation model (Turner et al., 1994) with a twinning scheme (Clausen et al., 2008) and extended to finite strains (Neil et al., 2010) is used for theoretical description of dislocation slip and mechanical twinning on the mesoscopic (grain) level. As described in the above cited works, the model uses the Eshelby inclusion approach (Eshelby, 1957) for description of interaction of each grain (sc. ellipsoidal elastoplastic inclusion), having a particular orientation, with the surrounding polycrystal treated as homogenous equivalent medium (HEM). The macroscopic applied stress and strain is accommodated by the elastic and plastic deformation at the grain level, where the condition for the activation of a particular slip or twinning system is governed by Schmid law, i.e. the resolved shear stress should exceed a critical value. Consequently, grains in "soft-orientation" can yield already at low stresses, whereas the "hard-oriented" grains respond elastically up to high applied stresses. Finally, for the evolution of the instantaneous CRSS $\tau_{s}$ with the total accumulated plastic strain $\Gamma$ within the grain an extended Voce hardening rule applies as follows:

$$
\tau^{s}=\tau_{0}^{s}+\left(\tau_{1}^{s}+\theta_{1}^{s} \Gamma\right)\left(1-\exp \left(-\frac{\theta_{0}^{s} \Gamma}{\tau_{1}^{s}}\right)\right),
$$


where $\tau_{0}^{s}$ and $\tau_{1}^{s}$ are the initial and back-extrapolated CRSS, respectively. Further fitting parameters are $\theta_{0}^{s}$ and $\theta_{1}^{s}$, i.e. the initial and asymptotic hardening rates. Since the original EPSC model is suitable primarily for characterization of slip-dominated deformation (Agnew and Duygulu, 2005), it was extended by Clausen et al. (Clausen et al., 2008) in order to account for twin domain reorientation and associated stress relaxation. The model assumes nucleation of multiple twin orientations within a single grain and permits the consuming of the parent by twin. The nucleated twins are treated as new grains, having a well-defined crystallographic orientation with respect to their parent grain. The twins are allowed to grow, but the total volume of the parent and the twins is kept constant. The model assumes nucleation of multiple twin orientations within a single grain and permits the complete consumption of the parent by twin. The backstress acting between the parent and twin is also considered and used for estimation of the twincaused stress relaxation in the matrix (for details see p. 2459 in (Clausen et al., 2008)).

\section{Results and discussion}

\subsection{Acoustic emission data analysis}

Raw AE signals recorded during tension and compression in the threshold-less regime (waveform streaming) are presented in Fig. 2. The AE streams appear as time-series of burst signals with various amplitudes arriving randomly in time. The typical lenticular shape of the stream exhibiting a clear maximum indicates an uneven distribution of AE activity during straining. This effect is ascribed to the gradual reduction of the dislocation mean free path owing to the increasing dislocation density (Máthis et al., 2011). The difference in the AE response between the two loading modes is obvious. Distinct burst events are observable during the whole test in tension, whereas in compression the burst ceases to appear at higher strains. Two main $\mathrm{AE}$ sources have long been recognized in magnesium alloys: dislocation slip and twinning (Friesel and Carpenter, 1984; Heiple and Carpenter, 1987a, b). Recent studies (e.g. (Ispánovity et al., 2010)) indicate that macro-plasticity is realized through simultaneous breakaway of dislocations pinned at different places within the specimen. Since the continuum mechanics based calculations suggest that the $\mathrm{AE}$ signal emitted by a single dislocation is not detectable by conventional means (Heiple and Carpenter, 1987a) it is evident that the dislocation-type AE is caused by dislocation avalanches (Lebyodkin et al., 2013) consisting of cooperatively moving dislocation segments. This process is activated concurrently in many grains, thus giving rise to overlapping in $\mathrm{AE}$ 

nucleation is associated with an abrupt motion of strongly correlated twinning dislocations. Consequently, the twinning AE signals are high amplitude transients. The AE events originated from twinning usually have higher amplitudes and power than the dislocation ones. Nevertheless, owing to the concurrency of the processes the visual inspection rarely leads to satisfactory results. In our recent paper (Čapek et al., 2014), the conventional $k$-means analysis was applied to hitbased (i.e. extracted by threshold) AE data. It was shown that both dislocation slip and twinning are active during straining, but the results describe only a general trend and a detailed analysis is missing. As it will be shown below, the ASK analysis applied here characterizes the dynamics of the system with significantly higher time-resolution and can distinguish not only between slip and twinning but it is also sensitive to activation of different slip systems.

As it is indicated above, the ASK analysis of waveform streaming data includes the following crucial steps:

- The data are sectioned into consecutive frames. In our case each frame contains 2048 samples which means that a frame correspond to a $0.002 \mathrm{~s}$ "time window".

- Power spectral density (PSD) function is calculated for each frame.

- The features of the PSD in the first frame define Cluster \#1.

- PSDs in the consecutive frames are analyzed one-by-one. If the statistical properties of a given PSD are similar to those in an already existing cluster, this PSD is assigned to this cluster. If not, a new cluster is established. The conditions for new cluster forming are based on $k$-means method.

- When the clustering procedure is completed, a dominant AE source mechanism is assigned to each cluster, based on the characteristic features (e.g. energy, frequency distribution etc.) of the PSDs (more details see below).

\section{Characteristic PSDs of the particular clusters}

The classification of AE signals based on quantitative comparison of their Fourier power spectra reveals that all signals fall naturally into five categories having specific, statistically different PSDs. Average PSD functions for each cluster identified in tension are presented in Fig. $3^{1}$. Their interpretation requires keeping in mind the fact that the final shape is significantly influenced by

\footnotetext{
${ }^{1}$ Since the PSDs in compression look very similar, they are not presented here.
} 
both the used AE transducer and the specimen. The 'broadband' feature of the transducer is achieved by dumping several resonant peaks that causes uneven distribution of sensitivity in frequency domain. The local maxima in all PSDs around $150 \mathrm{kHz}$ and $450 \mathrm{kHz}$ (upper harmonics) are caused by this effect. Furthermore, the specimens have their own resonant frequency, resulting in a distinct peak around $380 \mathrm{kHz}$.

\section{$\underline{\text { Results of the clustering procedure }}$}

Despite the very similar visual appearance of PSDs, the clusters are statistically distinct. We obtained stable and consistently defined clusters by application of $3 \sigma$ criterion. So-called crossplots, when two characteristic parameters of PSDs are plotted on axes $x$ and $y$, are the most suitable form for presenting the results of the cluster procedure. In Fig. 4 such an energy-mean frequency cross-plot is shown. The colors represent the particular clusters and $95 \%$ confidence ellipses added in order to highlight the difference between the respective populations of signals. Some overlapping of clusters is comprehensible, since such a plot represents a 2D projection of a 7-dimensional space.

The results of clustering can be plotted also in 3D, as it is presented in Fig. 5. In this case, we choose the following representation of the data:

- The kurtosis of PSD functions and the mean frequency are plotted on axes $x$ and $y$, respectively.

- The elements in the particular clusters are sorted out based on their energy into:

a) low energy ( $\mathrm{E}_{\mathrm{low}}<5 \times 10^{-4}$ a.u.) $\mathrm{AE}$ events

b) medium energy $\left(5 \times 10^{-4}\right.$ a.u. $<\mathrm{E}_{\text {medium }}<.5 \times 10^{-3}$ a.u. $) \mathrm{AE}$ events

c) high energy $\left(\mathrm{E}_{\text {high }}>5 \times 10^{-3}\right.$ a.u. $)$ AE events.

Afterwards we counted the number of events in the above defined energy ranges as a function of the values of kurtosis and mean frequency, in accordance with the cluster classification. This "number of events" is plotted on axis $z$. It is obvious from Fig. 5a that only the clusters 1 (noise $^{2}$ ) and 3 (non-basal slip) are in the low energy range. Nevertheless, Fig. 5b shows, that cluster 3 contains significant number of medium energy signals, as well as cluster 2 (basal slip). Majority of signals in cluster 4 and 5 belongs to the high energy range (Fig. 5c). It should be noted that only the compression data are plotted in Fig. 5, since the tensile data are less illustrative.

\footnotetext{
${ }^{2}$ Dominant AE mechanism in the cluster - for explanation see the next section.
} 


\section{Determination of dominant AE source mechanisms in the particular clusters}

The dominant AE source mechanisms in the particular clusters are listed in Table 2. They can be determined using a closer inspection of characteristic features of the clusters' elements. Further indicator is the strain (time) evolution of cumulative (i.e. gradual increment) number of events in the particular clusters (Fig. 6) and the corresponding cumulative energies (Fig. 7).

Table 2 - Dominant AE source mechanisms in particular clusters

\begin{tabular}{|l|l|l|}
\hline Cluster \# & Color code in Figs. 5 and 6 & Source mechanism \\
\hline 1 & Grey & Noise \\
\hline 2 & Red & Basal slip \\
\hline 3 & Orange & Non-basal slip \\
\hline 4 & Blue & Extension twinning 1 \\
\hline 5 & Magenta & Extension twinning 2 \\
\hline
\end{tabular}

It is evident that Cluster 1 includes the background noise signals, since it appears before the onset of deformation test and has a low energy. Furthermore, Cluster 1 exhibits broad frequency spectrum, which is typical for the background noise (Pomponi and Vinogradov, 2013).

The remaining four clusters unambiguously have a microstructural origin. Similar to other authors (Dobroň et al., 2007; Lou et al., 2007); (Vinogradov et al., 2013b) we observed activity of deformation mechanisms far below the macroscopic yield point.

The beginning of straining is accompanied by the appearance of Cluster 2 (Fig. 6a). The events have medium energy (cf. Fig. 5b). Above the macroscopic yield point number of elements in Cluster 3, having low and medium energies (Fig. 6a) increases significantly. If we have a look on the waveforms of these elements (Fig. 6a), they exhibit a continuous character, which indicates a dislocation origin of the clusters.

The number of elements in the Clusters 4 and 5 starts to increase at slightly higher stresses (Fig. $6 b$ ), but the energy of these events is larger than that for clusters 2 and 3 (Figs. 5c and 7). This behavior refers to the nucleation of extension twins, which have higher energy $\mathrm{AE}$ response (Heiple and Carpenter, 1987b) and the CRSS for their activation is slightly larger than that for 


\footnotetext{
${ }^{3}$ In this context the twin propagation means the growth of the twin in length. The authors are convinced that such twin propagation is the source of the AE. On contrary, the AE of twin growth, i.e. thickening in width is not detectable (see text).
} be noted that the ASK analysis does not preclude the possibility of concurrent activity of various deformation mechanisms. The method only tells us which deformation mechanism dominates at a given strain (time) level.

The dislocation slip generating the signals from Cluster 2 is activated at low applied stresses. This behavior is characteristic for the basal $\langle a\rangle$ slip, which has the lowest CRSS (Akhtar and Teghtsoonian, 1968; Chapuis and Driver, 2011). The common feature of the dislocation slip related to Cluster 3 in both tension and compression is that its activity dramatically increases above the macroscopic yield point. As it will be presented in sections 3.2 and 3.3, this behavior can be associated with the activation of the prismatic $\langle a\rangle$ slip, having higher CRSS than the basal system. Its weak activity at the onset of the straining is most probably connected with the initial dislocation configuration, as it will be discussed in section 3.2. The evolution of both basal and prismatic slip is slightly different for the particular deformation modes: in tension, the basal slip maintains its significant role in deformation throughout the test, even after the onset of prismatic $\langle a\rangle$-slip. In compression, the basal slip is suppressed, once the non-basal slip is activated (see Fig. 6a). Furthermore, in tension the non-basal slip become active later (at higher strains) than in compression. This result can be rationalized in terms of a smaller volume fraction of grains which are well-oriented for twinning (Agnew et al., 2001; Cáceres and Blake, 2007) during tension, as well as the necessity of accommodation of deformation by dislocation slip once twinning is completed.

The distribution of the twin-related signals into two clusters is most probably connected with the twin size dependence of the PSD. At the beginning, the twins nucleate in 'virgin' grains, where they can propagate ${ }^{3}$ quickly from one grain boundary to the other on the opposite side. As the strain increases, nucleation of further twin generations in the same grain is necessary to accommodate the local strain. Nevertheless, the path for twin propagation is limited by the already existing twins. Consequently, the elastic energy released by twin is smaller and the shape of the PSD changes. The dependence of the twinning clusters on the loading mode is also 
evident. In tension, both twinning-associated AE clusters monotonically grow with time (strain), whereas in compression the supremacy of the twin events terminates shortly after reaching the yield point. The detailed analysis of this phenomenon was reported in our concurrent work (Čapek et al., 2014). Therefore, only a short notice is given here. The twinning mechanism can be sorted out into two stages: nucleation and growth. The twin nucleation is a quick mechanism with a characteristics speed of about $4400 \mathrm{~m} / \mathrm{s}$ which is in the transonic regime (Finkel et al., 1970; Gumbsch and Gao, 1999). Therefore, the emitted AE has a high power and is well detectable. On contrary, the motion of the twin boundary associated with twin thickening is slow (its speed is in the order of $10^{-6} \mathrm{~m} / \mathrm{s}$ ) and therefore the twin growth is undetectable by AE (Papirov et al., 1984). Bearing this in mind, we can conclude that, in tension, the twin nucleation persistently occurs during straining, whereas in compression the twin nucleation is negligible at higher strains. At this point, it is timely to recall the neutron diffraction results, which show a monotonic increase of the twin volume for both loading modes (Čapek et al., 2014). This is possible only in the case when in compression a rapid twin growth takes place, as it is discussed in Section 3.3.

\subsection{CMWP fitting of the neutron diffraction pattern}

As an example, the fitting of the diffractogram detected for the initial sample and deformed by compression up to the strain of $6 \%$ is shown in Fig. 8. The open circles and the solid line represent the measured data and the fitted curves, respectively. Fig. 9a illustrates the classical Williamson-Hall plot for the sample deformed by compression up to $6 \%$. In this figure, the full width at half maximum (FWHM) is plotted as a function of the length of the diffraction vector $(g)$. The instrumental peak width was subtracted from the breadth of the Mg line profiles assuming a linear addition rule for the instrumental and microstructural broadening effects. It should be noted that this rule is strictly valid only when both the instrumental and the microstructural profiles have Lorentzian shape, which in the case of the experimental curves might not be completely fulfilled owing to the experimental error. However, this method of the instrumental correction for the peak breadth does not call into question the reliability of the present Williamson-Hall analysis, as only qualitative conclusions will be drawn from the plots.

The line broadening in Fig. 9a is very anisotropic, i.e. it varies non-monotonously with increasing the length of the diffraction vector. The $h k l$-dependence of the diffraction peak broadening in plastically deformed metals is usually caused by the anisotropic strain field of dislocations (Ungár \& Borbély, 1996). In this case the FWHM values can be arranged along a smooth 
monotonous curve by plotting them as a function of $g\left(\bar{C}_{h k l}\right)^{1 / 2}$ (referred to as modified Williamson-Hall plot). As an example, Fig. 9b shows the modified Williamson-Hall plot for the sample deformed by compression up to $6 \%$.

The dislocation density as a function of strain for tension and compression is shown in Fig. 10. The dislocation density increases with increasing strain for both tension and compression. For strains up to $2 \%$ the dislocation densities obtained for tension and compression agree very well. However, at 4 and $6 \%$ the dislocation density in the compressed sample is slightly larger. According to our opinion the evolution of the dislocation density is significantly influenced by the twinning. The AE results, the ND and EPSC data (see Section 3.3) indicate a rapid twin growth and higher volume fraction of twins in compression, hence a larger contribution to strain accommodation. The dislocation density involves both accumulation and recovery terms. It seems that the recovery term is smaller in compression. This feature can be discussed in terms of dislocation transmutation upon the passage of a twin front (Niewczas, 2007; El Kadiri and Oppedal, 2010). El Kadiri has shown that if the $\langle a\rangle$ dislocations cross the twin boundary, creation of sessile dislocations within the twins can be expected. (e.g. $\langle c\rangle$-types, but they don't necessarily belong to a usual slip system). Thus, the annihilation of these dislocations is hindered. Further, the theoretical models predict a higher activity of $\langle c+a\rangle$ dislocations during compression, particularly in twins (Agnew and Duygulu, 2005). Some experimental evidence of enhanced density of $\langle c+a\rangle$ dislocations was found also using transmission electron microscopy (TEM) (Bhattacharyya et al., 2009). Thus, the reduced dislocation annihilation and facilitated $\langle c+a>$ slip can result in a higher dislocation density in compression at higher strains.

In order to verify experimentally the theory above, the fractions of the different dislocation slip system families were determined from parameters $q_{1}$ and $q_{2}$ using the procedure described in section 2.2. It was found that in the initial sample there was an abundance of <a>-type dislocations (about 77\%), while the fraction of $\langle c+a\rangle$ dislocations was about $18 \%$ and the amount of $\langle c\rangle$ dislocations was small (5\%). In the $\langle a\rangle$-type Burgers vector group the majority of dislocations are prismatic or pyramidal edge. The screw fraction in $\langle a\rangle$-type dislocations was about $3 \%$ in the initial case. Fig. 11 shows that the ratio of basal to prismatic $\langle a\rangle$-type dislocations further decreased both during tension and compression at the strains of 4 and $6 \%$. It is noted that the $\mathrm{AE}$ results are in agreement with this observation: the number of non-basal slip origin AE signals rapidly increases above the strain of $4 \%$ (c.f. Fig. 6). Unlike the radial 
detectors, where there is no significant difference in the evolution of the basal/prismatic $\langle a\rangle$ dislocation ratio for the particular loading modes, in the signal of the axial detector larger fraction of prismatic $\langle a\rangle$ dislocations was found for tension. Furthermore, in tension, the ratio of $\langle a\rangle$ and $\langle c+a\rangle$ dislocations did not change significantly with increasing strain (see Fig. 11a,b). In contrast, in the case of compression the diffractogram in the radial detector suggests that the fraction of $\langle a\rangle$ dislocations increased with increasing strain up to $6 \%$ at the expense of $\langle c+a\rangle$ dislocations (see Fig. 11c) while in the axial detector increased fraction of $\langle c+a\rangle$ dislocations was detected. The opposite evolution of the $\langle a\rangle /\langle c+a\rangle$ ratio in the particular detectors is most probably a consequence of the quick twin growth during compression and the applied diffraction geometry. The direction of the axial detector is designated in twinning as in all volumes with the highest twinning Schmid factors the c-axes rotate to this direction. Therefore, the axial detector is very sensitive to the twinning process. Owing to the averaging effect in radial detector the contribution of the twins is less pronounced than that in the axial detector. Furthermore, in compression there are many grains with high twinning Schmid factor in which only one or two twin variants are formed. These twins can rapidly grow with increasing strain (Beyerlein et al., 2010; El Kadiri and Oppedal, 2010; Čapek et al., 2014)) and they significantly contribute to the (00.l) peaks in the axial detector, as indicated by the texture (see Fig. 13 in Section 3.3). In twins higher density of $\langle c+a\rangle$ dislocations is expected owing to the higher Schmid factor for $2^{\text {nd }}$ order pyramidal slip in these orientations (Muránsky et al., 2010a). In addition, a significant part of $<a>$-dislocations can be transmuted in the twins into other dislocation types due to the interaction with twin boundaries (Oppedal et al., 2012b), while $<c+a>$-dislocations can pass through the twin boundaries without any transmutation (Mendelson, 1970). As mentioned above, significant part of $\langle a\rangle$-dislocations can transmute in the twins for different dislocation types. Thus, the fraction of $\langle c+a\rangle$ dislocation increases in the axial detector at the expense of $\langle a\rangle$ dislocations, as it is obvious from Fig. 11d.

In the radial detector, besides the twinned volumes the parent grains also have a significant contribution to the diffraction pattern. The $\langle a\rangle$-type dislocations in parents were found strongly influenced by twin growth: i) the twin boundaries can act as barriers to slip dislocations ((Serra et al., 1999); (Capolungo et al., 2009); ii) dissociation of twinning dislocations can lead to formation of $\langle a\rangle$ dislocations ((Mendelson, 1970). Both these mechanisms increase density of $<a\rangle$ dislocations in parent grains (Fig. 11c). 
In the case of tension, the strain in high twinning Schmid-factor grains is accommodated by nucleation of several twin variants (Čapek et al., 2014). Since the growth of these twins is limited (Yu et al., 2014) the above discussed process is not so significant. In the axial detector, which the $\langle a\rangle\langle\langle c+a\rangle$ ratio remains unchanged within the experimental error, whereas in radial detector slightly decreases.

\subsection{EPSC modeling predictions and their comparison with the neutron diffraction results}

The present EPSC modeling incorporated 20000 grains with random texture distribution. Based on previous papers (Agnew et al., 2006; Clausen et al., 2008) the following four deformation mechanisms were considered in the calculations: the $\langle a\rangle$ slip in $\langle 11 \overline{2} 0\rangle$ direction on (0001) basal and $\{10 \overline{1} 0\}$ prismatic planes, second-order $(\{11 \overline{2} 2\}\langle\overline{1} \overline{1} 23\rangle)$ pyramidal $\langle c+a\rangle$ slip and $\{10 \overline{1} 2\}-$ type extension twinning. Since the maximum measured strain level was only $6 \%$, the $\{10 \overline{1} 1\}-$ type compression twinning - usually observed at higher strains (Agnew et al., 2013) - was not included in the analysis. The initial CRSS and hardening values used to fit the predicted macroscopic stress-strain curves to the experimental ones are listed in Table 3. It is obvious from Fig. 12 that the coincidence between the calculated and measured curves is satisfying. The loading mode dependence of twinning indicated by AE results must significantly influence the development of the deformation texture. The inverse pole figures (IPF) at $0 \mathrm{~h}, 3$ and $6 \%$ deformation for radial detector are shown in Fig. 13. In compression, the intensity increases at 0002 pole with increasing strain in axial detector and a high intensity is evident at $10 \overline{1} 0$ pole in radial detector, respectively.

Table 3 - Initial fitting parameters of EPSC model, based on Eq. 1

\begin{tabular}{|l|l|l|l|l|}
\hline & $\tau_{0}{ }^{\mathrm{s}}[\mathrm{MPa}]$ & $\tau_{1}{ }^{\mathrm{s}}[\mathrm{MPa}]$ & $\theta_{0}{ }^{\mathrm{s}}[\mathrm{MPa}]$ & $\theta_{1}{ }^{\mathrm{s}}[\mathrm{MPa}]$ \\
\hline Basal $\langle a\rangle$ & 4 & 2 & 200 & 125 \\
\hline Prismatic $\langle a\rangle$ & 19 & 8 & 250 & 100 \\
\hline Pyramidal $\langle c+a\rangle$ & 75 & 60 & 300 & 150 \\
\hline
\end{tabular}


This is a clear signature of rapid texture evolution due to extension twinning. In tension, a similar behavior is apparent, but the IPFs in the particular detectors behave exactly in opposite way due to the polar nature of the twinning. The maxima are approximately two times higher in compression, which is in accordance with the assumption of rapid twin growth in this loading mode. The predicted textures are in good agreement with the measured ones for all strain levels. Since in the implemented model the texture development is attributed to the extension twinning, these results prove its major role in the deformation process.

The comparison of the calculated twin volume fraction with the measured ${ }^{4}$ one (taken from (Čapek et al., 2014)) is presented in Fig. 14. In accordance with the texture development, the twinned volume in tension is smaller. The EPSC model slightly underestimates the experimental values, but the course of the calculated curves follow the measured data points well. The strain evolution of the number of nucleated twin variants, predicted by EPSC model (Fig. 15) and the evolution of the twin induced AE events (cf. Fig. 6) are in good agreement. In compression, the number of the nucleated twin variants within a single grain increases with decreasing Schmid factor (TwSF) for twinning (Beyerlein et al., 2010). Since in compression there are more grains having high TwSF $(>0.4)^{5}$ (Cáceres and Blake, 2007) and therefore lesser nucleated twin variants is expected in conjunction with Fig. 15, where the number of nucleated twin variants per grain is shown. The same feature is presented in Fig. 16a, where in the ideally oriented grain (i.e. $c$-axis is perpendicular to the loading direction) marked as $\mathrm{C} 1$, only the twin variants having the largest TwSF $(=0.5)$ are nucleated. In the grain $\mathrm{C} 2$, deviated from the ideal orientation by approx $27^{\circ}$, four variants can be observed. It should be noted that if the parent grain departs from its ideal orientation by more than $\sim 46^{\circ}$, the twinning in compression is not feasible (Čapek et al., 2014). In tension, there is an opposite trend. As it is evident from Fig. 16b, all six twin variants can be nucleated within an ideally oriented grain (T1), where the $c$-axis is aligned with the loading direction. As it is shown in our current paper (Čapek et al., 2014), in tension the plastic deformation is not conceivable without twinning in low TwSF grains. Thus twinning takes place in larger $c$-axis vs. loading axis angle range (up to $\sim 64^{\circ}$ ), which can be seen also in Fig. 16c,

\footnotetext{
${ }^{4}$ The twin volume fraction was calculated from the changes in the area under the axial distribution function of the (00.2) peak (Čapek et al., 2014). For further details see (Clausen et al., 2008).

${ }^{5}$ In this case the twin variant with the highest Schmid factor in a given grain is meant
} 
where in grains $\mathrm{T} 2$ and $\mathrm{T} 3$, twin variants having TwSF 0.04 and 0.01 , respectively were nucleated. For such orientations four from six possible twin variants have negative TwSF. Thus there nucleation is not plausible. This means that in tension the number of nucleated twin variants decreases with decreasing TwSF.

In summary, the higher number of nucleated twins in tension can be rationalized in terms of the nucleation of several different twin variants in grains having orientation close to the optimal. The twin growth is hindered in these grains, most probably due to the twin-twin interactions which lead to formation of basal-basal and prism-prism tilt boundaries (Yu et al., 2014). In contrast, in compression, where it is a large number of ideally oriented grains with only one or two twin variants, the twin growth is easier and twinned volume fraction is larger. This result is consistent also with AE findings in Section 3.1 (twin nucleation AE events: monotonically increasing in tension vs. terminated soon above macroscopic yield in compression).

The variations of the relative activity of each deformation process for both loading modes versus the applied stress obtained by EPSC modeling are depicted in Fig. 17. In tension, there is a massive onset of basal slip and extension twinning already at low applied stresses. In the vicinity of the macroscopic yield point the prismatic $\langle a\rangle$ slip activates and exhibits a monotonically increasing behavior. The activity of the basal slip gradually decreases, most probably due to the decreasing volume of the favorable oriented grains. The activity of the second-order pyramidal slip is negligible. In compression, the macroscopic yielding is also associated with the decrease of the activity of twinning while the role of basal and non-basal slip increases. There are two remarkable differences with respect to the tension results. First of all the prismatic $\langle a\rangle$ slip is not so pronounced as in tension at higher stresses, secondly above the strain of $4 \%$ the contribution of $\{11 \overline{2} 2\}\langle\overline{1} \overline{1} 23\rangle$ pyramidal slip slightly increases. The predicted CRSS for pyramidal slip is in good agreement with the experimental values (Yoshinaga and Horiuchi, 1964).

The comparison of the calculated and measured elastic lattice strains is depicted in Fig. 18. The non-linear behavior of the experimental internal strain data is qualitatively quite well predicted. The model satisfactorily describes the relaxation of lattice strains on $\{0002\}$ and $\{10 \overline{1} 3\}$ planes as well as the load accumulation of the $\{10 \overline{1} 0\}$ and $\{11 \overline{2} 0\}$ lattice strains in radial direction in compression and in axial direction in tension. These reflections have parent $(\{0002\}$ and $\{10 \overline{1} 3\})$ and daughter $(\{10 \overline{1} 0\}$ and $\{11 \overline{2} 0\})$ orientation for extension twinning with respect to these detectors, so they characterize this deformation mechanism. If we interchange the parent- 


\section{Discussion}

In the present work a large number of various theoretical (EPSC) and experimental (AE, ND) results are presented. In order to have a clear overview about the agreement between the various outputs, we directly compared the particular datasets:

\subsection{EPSC vs. AE}

In order to get a better basis for comparison of EPSC and AE results, in Fig. 19 we plot the "relative AE source activity (RSA)" against the applied stress together with the EPSC results from Fig. 17. $\mathrm{RSA}^{6}$ describes the proportion of particular AE sources at the given stress level. For right interpretation of Fig. 19 it should be kept in mind that ASK method determines the dominant AE source mechanism in a given time window (see §3.1). It means that in the case when a given $\mathrm{AE}$ source is dominant for longer period (i.e. in several consecutive time (stress) windows), the contributions of other sources decrease to zero. On contrary, the relative system activity, calculated using EPSC model can handle the concurrently active deformation mechanisms. Thus, the evolution of the activities calculated by EPSC and AE may slightly differ. The AE method cannot make difference between the prismatic and $2^{\text {nd }}$ order pyramidal slip. Thus, for sake of simplicity, only the three major deformation mechanisms: basal slip, non-basal slip and twinning are plotted in Fig. 19.

Basal slip - both methods show activation of the basal slip in the early stage of straining. In tension (Fig. 19a) the decreasing trend of activity of basal slip above $50 \mathrm{MPa}$ is obvious from both EPSC and AE results. In compression (Fig. 19b) the increment of basal activity between 30

\footnotetext{
${ }^{6}$ The calculation of the RSA was based on the data in Fig. 6 .
} 
and $40 \mathrm{MPa}$ can be seen. The EPSC at higher stresses in compression indicates a constant activity of basal slip, whereas the AE shows a nearly zero value. This effect can be explained by two reasons: i) owing to the intensive slip, the dislocation density in basal plane increases, which shorten the mean free path for dislocation glide and consequently the basal AE signal weakens; ii) since in compression besides the prismatic $\langle a\rangle$-slip the $\langle c+a\rangle$-slip is also active, the contribution of non-basal slip to $\mathrm{AE}$ frequency spectra dominates at higher stresses. Consequently, the fraction of "basal AE" decreases to zero (see comment above).

Non-basal slip - both modeling and AE experiment indicate dominant role of non-basal slip at higher stresses. The non-basal slip in AE starts to be dominant later in comparison to EPSC, since around the yield point the high energy twinning signals "talk down" the lower dislocation ones. In tension (Fig. 19a) the AE results nicely show, how the activity of the non-basal slip become dominant above $60 \mathrm{MPa}$. In compression the non-basal activity shows an opposite trend above 60 $\mathrm{MPa}$. The EPSC result exhibits slight increment, whereas the AE results reveal a decrease. At such a high stresses in compression: i) twin growth takes place, which gives no AE signal; ii) AE response of basal slip is weak (see above); iii) the majority of non-basal AE events is a low energy one (see Fig. 5a). Therefore, the noise signal (not plotted in Fig. 19) can dominate above the non-basal AE, which causes the decreasing of the proportion of the latter.

Twinning - both EPSC and AE indicates onset of extension twinning at low applied stress. In the range of 15-40 $\mathrm{MPa}$ in tension and 10-30 $\mathrm{MPa}$ in compression the twinning dominates over the all other AE source mechanisms owing to its large magnitude (cf. Fig. 5c). In tension the trend of the EPSC and AE activity is in agreement. In compression the $\mathrm{AE}$ activity of twinning is close to zero above $30 \mathrm{MPa}$, which simply reflects the fact that the $\mathrm{AE}$ is not sensitive for twin growth, whereas EPSC takes this mechanism into account.

\subsection{EPSC vs. CMWP}

In this case it should be kept in mind that modeling gives the slip system activity according to the Schmid's law while neutron diffraction describes the slip system populations, i.e. the former method compares the easiness of the glide in the different slip systems while the latter procedure characterizes the density of various types of dislocations. For instance, EPSC calculation does not take directly into account the abundance of non-basal < $a\rangle$-type dislocations relative to basal dislocations in the initial state. Thus, the reduction of the basal slip activity during deformation 


\subsection{Further remarks}

The results presented above nicely demonstrate, how a combination of theoretical and experimental methods can lead to profound characterization of deformation processes in magnesium. However, this approach has a potential for deriving more perspicacious connection between the microstructure and the processes of the plastic deformation. Since a deeper analysis is out of scope of this paper, only a short notice about the further possible actions is given here.

As it was presented recently by Vinogradov et al. (Vinogradov et al., 2014), the median frequency $f_{m}$ is bound with the dislocation density. For face centered cubic metals the relation 
$f_{m} \sim \sqrt{\rho}$ was found as long as the plastic deformation is uniform (Vinogradov et al., 2014). Such an analysis can be performed also for this dataset, since the ND results offers a possibility of direct verification of the above mentioned relation.

The modeling results can also be refined. The original EPSC model works with the assumption that the strain and stress within a grain are uniform. Therefore, the intragranular effects cannot be directly included into the EPSC framework. From the point of view of ND results this would be desirable, since these, so-called Type III stresses contribute to peak width changes. A self-consistent model including dislocation hardening was worked out by Beyerlein and Tomé (Beyerlein and Tomé, 2008). They gave the evolution of the dislocation density as:

$$
\frac{\partial \rho^{\alpha}}{\partial \gamma^{\alpha}}=\frac{\partial \rho_{g e n}{ }^{\alpha}}{\partial \gamma^{\alpha}}-\frac{\partial \rho_{d e b}{ }^{\alpha}}{\partial \gamma^{\alpha}}-\frac{\partial \rho_{a n n}{ }^{\alpha}}{\partial \gamma^{\alpha}},
$$

where $\rho_{\text {gen }}$ means density of generated dislocations, $\rho_{\text {deb }}$ is dislocation density locked in debris and $\rho_{a n n}$ is the density of the annihilated dislocations, and index $\alpha$ reflects the particular slip modes. They used further the Mecking-Kocks (Mecking and Kocks, 1981) evolution model for dislocation density:

$$
\frac{\partial \rho^{\alpha}}{\partial \gamma^{\alpha}}=k_{1}^{\alpha} \sqrt{\rho^{\alpha}}-k_{2}^{\alpha}(\dot{\varepsilon}, T) \rho^{\alpha},
$$

where the coefficients $k_{1}$ and $k_{2}$ represent the rate of storage of gliding dislocations at the dislocation forest obstacles or dislocation cell walls, and the rate of dynamic recovery, respectively.

Initial slip resistance, which depends on the slip mode $\alpha$, work hardening due to interaction of dislocations with forest dislocations and debris, and twin boundary-dislocations interactions were taken into the account as the main contributors to slip resistance. The model was successfully used for describing the deformation behavior of high-purity $\mathrm{Zr}$ (Beyerlein and Tomé, 2008). The first attempt for comparing the output of this dislocation-density based model with ND results measured on extruded magnesium alloy was published by Clausen et al. (Clausen et al., 2010) and recently by Wen et al. (Wen et al., 2014). The results were promising, but a necessity of further development of both experimental and theoretical parts was concluded. A possible way for this development has been shown by Ahn et al. (Ahn et al., 2012), who extends the original Kocks-Mecking model with an additional storage term associated with the twin spacing. 


\section{Conclusions}

The loading mode dependence of deformation mechanisms in a pure, randomly oriented magnesium polycrystal was investigated at room temperature using various in-situ experimental (AE, ND) and computational (EPSC) methods. The maximum applied strain was 6\%. It can be concluded that the particular results form a complementary dataset, which helps in getting a more precise description of the plastic deformation processes.

Generally, the novel potential of AE and ND experimental methods was revealed:

- $\mathrm{AE}$ - using a proper analysis of raw $\mathrm{AE}$ waveform streaming dataset, high-time resolution information about the dynamics of active deformation processes can be derived. It is shown that the method is capable to make difference not only between dislocation slip and twinning AE events, but also between basal and non-basal slip events, and various twinning mechanisms.

- $\mathrm{ND}$ - in combination with CMWP evaluation procedure, quasi in-situ information can be obtained about the fraction of dislocations in various slip systems. The evaluation method is capable for revealing the evolution of density of $\langle c+a\rangle$, as well as basal and non-basal $\langle a\rangle$ dislocations with applied stress, which can be hardly realized with such a good statistics using other experimental methods.

Concerning the deformation mechanisms during tensile and compression tests in magnesium polycrystal, the following conclusions can be drawn:

- Both EPSC calculations and AE experiments indicate a dominant role of the basal slip and extension twinning at the onset of straining.

- However, the macroscopic yield is accompanied by a considerable increase of the fraction of non-basal $\langle a\rangle$-type dislocations, as it was shown by AE and ND experiments.

- Difference was found in the activity of $\langle c+a\rangle$-slip, which is more significant in compression.

\section{Acknowledgement}

The authors are grateful for the financial support of the Czech Science Foundation under the contract 14-36566G. JČ acknowledges the support from the Grant Agency of Charles University. JG acknowledges the support form the Hungarian Scientific Research Fund, OTKA, Grant No. 
K-109021. AV acknowledges the support from the Russian Ministry of Education and Science through the grants-in-aid 11.G34.31.0031 and 14.583.21.0006

This work has benefited from the use of the Lujan Neutron Scattering Center at LANSCE, funded by the US Department of Energy's Office of Basic Energy Sciences. Los Alamos National Laboratory is operated by Los Alamos National Security LLC under US DOE Contract DEAC52-06NA25396.

\section{References}

Agnew, S.R., Brown, D.W., Tomé, C.N., 2006. Validating a polycrystal model for the elastoplastic response of magnesium alloy AZ31 using in situ neutron diffraction. Acta Mater. 54, 4841-4852.

Agnew, S.R., Duygulu, O., 2005. Plastic anisotropy and the role of non-basal slip in magnesium alloy AZ31B. Int. J. Plast. 21, 1161-1193.

Agnew, S.R., Mulay, R.P., Polesak, F.J., Calhoun, C.A., Bhattacharyya, J.J., Clausen, B., 2013. In situ neutron diffraction and polycrystal plasticity modeling of a $\mathrm{Mg}-\mathrm{Y}-\mathrm{Nd}-\mathrm{Zr}$ alloy: Effects of precipitation on individual deformation mechanisms. Acta Mater. 61, 3769-3780.

Agnew, S.R., Yoo, M.H., Tomé, C.N., 2001. Application of texture simulation to understanding mechanical behavior of $\mathrm{Mg}$ and solid solution alloys containing Li or Y. Acta Mater. 49, 42774289.

Ahn, D.H., Kim, H.S., Estrin, Y., 2012. A semi-phenomenological constitutive model for hcp materials as exemplified by alpha titanium. Scripta Mater. 67, 121-124.

Akhtar, A., Teghtsoonian, E., 1968. Solid Solution Strengthening in Alloy Single Crystals of Mg. Jom-J. Min. Met. Mat. S 20, A73-79.

Akhtar, A., Teghtsoonian, E., 1969. Solid Solution Strengthening of Magnesium Single Crystals .I. Alloying Behaviour in Basal Slip. Acta Metall. Mater. 17, 1339-1349.

Avedesian, M.M., Baker, H., 1999. Magnesium and Magnesium Alloys (ASM Specialty Handbook). ASM International, Materials Park.

Balík, J., Lukáč, P., Kužel, R., 2012. Basal to Non-Basal Transition for In-Plane Deformation of AZ31 Magnesium Alloys. Acta Phys Pol A 122, 435-438.

Barnett, M.R., Stanford, N., Ghaderi, A., Siska, F., 2013. Plastic relaxation of the internal stress induced by twinning. Acta Mater. 61, 7859-7867.

Beyerlein, I.J., Capolungo, L., Marshall, P.E., McCabe, R.J., Tomé, C.N., 2010. Statistical analyses of deformation twinning in magnesium. Philos. Mag. 90, 2161-2190.

Beyerlein, I.J., Tomé, C.N., 2008. A dislocation-based constitutive law for pure Zr including temperature effects. Int. J. Plast. 24, 867-895.

Bhattacharyya, D., Cerreta, E.K., McCabe, R., Niewczas, M., Gray, G.T., Misra, A., Tomé, C.N., 2009. Origin of dislocations within tensile and compressive twins in pure textured $\mathrm{Zr}$. Acta Mater. 57, 305-315.

Bourke, M.A.M., Dunand, D.C., Ustundag, E., 2002. SMARTS - a spectrometer for strain measurement in engineering materials. Appl. Phys. A 74, S1707-S1709.

Cáceres, C.H., Blake, A.H., 2007. On the strain hardening behaviour of magnesium at room temperature. Mater. Sci. Eng. A 462, 193-196. 
Cáceres, C.H., Lukáč, P., 2008. Strain hardening behaviour and the Taylor factor of pure magnesium. Philos. Mag. 88, 977-989.

Cáceres, C.H., Lukáč, P., Blake, A., 2008. Strain hardening due to $\{10-12\}$ twinning in pure magnesium. Philos. Mag. 88, 991-1003.

Capolungo, L., Marshall, P.E., McCabe, R.J., Beyerlein, I.J., Tomé, C.N., 2009. Nucleation and growth of twins in Zr: A statistical study. Acta Mater. 57, 6047-6056.

Clausen, B., Brown, D.W., Tomé, C.N., Balogh, L., Vogel, S.C., 2010. Engineering Related Neutron Diffraction Measurements Probing Strains, Texture and Microstructure, in: Hansen, N., Juul Jensen, D. (Eds.), 31st Riso International Symposium on Materials Science. Technical University of Denmark, Riso National Laboratory for Sustanaible Energy, pp. 11-29.

Clausen, B., Tomé, C.N., Brown, D.W., Agnew, S.R., 2008. Reorientation and stress relaxation due to twinning: Modeling and experimental characterization for Mg. Acta Mater. 56, 2456-2468. Čapek, J., Máthis, K., Clausen, B., Stráská, J., Beran, P., Lukáš, P., 2014. Study of the loading mode dependence of the twinning in random textured cast magnesium by acoustic emission and neutron diffraction methods. Mater. Sci. Eng. A 602, 25-32.

Dobroň, P., Bohlen, J., Chmelík, F., Lukáč, P., Letzig, D., Kainer, K.U., 2007. Acoustic emission during stress relaxation of pure magnesium and AZ magnesium alloys. Mater. Sci. Eng. A 462, 307-310.

Dobroň, P., Chmelík, F., Yi, S.B., Parfenenko, K., Letzig, D., Bohlen, J., 2011. Grain size effects on deformation twinning in an extruded magnesium alloy tested in compression. Scripta Mater. 65, 424-427.

Dragomir, I.C., Ungár, T., 2002. Contrast factors of dislocations in the hexagonal crystal system. J. Appl. Cryst. 35, 556-564.

El Kadiri, H., Oppedal, A.L., 2010. A crystal plasticity theory for latent hardening by glide twinning through dislocation transmutation and twin accommodation effects. J. Mech. Phys. Solids. 58, 613-624.

Eshelby, J.D., 1957. The Determination of the Elastic Field of an Ellipsoidal Inclusion and Related Problems, Proc. Roy. Soc. London A, 241, 376-396.

Fernandez, A., Prado, M.T.P., Wei, Y.J., Jerusalem, A., 2011. Continuum modeling of the response of a Mg alloy AZ31 rolled sheet during uniaxial deformation. Int J Plast. 27, 1739-1757. Finkel, V.M., Voronov, I.N., Savelyev, A.M., Yeliseyev, A.I., Fedorov, V.A., 1970. Hindering of Crack Propagations by Twins. Phys. Met. Metall. USSR 29, 131-139.

Fotouhi, M., Heidary, H., Ahmadi, M., Pashmforoush, F., 2012. Characterization of composite materials damage under quasi-static three-point bending test using wavelet and fuzzy C-means clustering. J. Compos. Mater. 46, 1795-1808.

Friesel, M., Carpenter, S.H., 1984. Determination of the Source of Acoustic Emission Generated during the Deformation of Magnesium. J. Acoust. Em. 6, 11-18.

Ghaderi, A., Siska, F., Barnett, M.R., 2013. Influence of temperature and plastic relaxation on tensile twinning in a magnesium alloy. Scripta Mater 69, 521-524.

Gharghouri, M.A., Weatherly, G.C., Embury, J.D., Root, J., 1999. Study of the mechanical properties of Mg-7.7at.\% Al by in-situ neutron diffraction. Philos. Mag. A 79, 1671-1695.

Gubicza, J., Máthis, K., Hegedűs, Z., Ribárik, G., Tóth, A.L., 2010. Inhomogeneous evolution of microstructure in AZ91 Mg-alloy during high temperature equal-channel angular pressing. J. Alloy. Compd. 492, 166-172.

Gumbsch, P., Gao, H., 1999. Dislocations faster than the speed of sound. Science 283, 965-968.

Hama, T., Takuda, H., 2011. Crystal-plasticity finite-element analysis of inelastic behavior during unloading in a magnesium alloy sheet. Int. J. Plast. 27, 1072-1092. 

Alloys - A Review: Part I. J. Acoust. Em. 6, 177-204.

Heiple, C.R., Carpenter, S.H., 1987b. Acoustic Emission Produced by Deformation of Metals and Alloys - A Review: Part II. J .Acoust. Em. 6, 215-237.

Hirsch, J., Al-Samman, T., 2013. Superior light metals by texture engineering: Optimized aluminum and magnesium alloys for automotive applications. Acta Mater. 61, 818-843.

Chapuis, A., Driver, J.H., 2011. Temperature dependency of slip and twinning in plane strain compressed magensium single crystals. Acta Mater. 59, 1986-1994.

Chmelík, F., Klose, F.B., Dierke, H., Sachl, J., Neuhäuser, H., Lukáč, P., 2007. Investigating the Portevin-Le Chatelier effect in strain rate and stress rate controlled tests by the acoustic emission and laser extensometry techniques. Mater. Sci. Eng. A462, 53-60.

Christian, J.W., Mahajan, S., 1995. Deformation twinning. Progress Mater. Sci. 39, 1-157.

Ispánovity, P.D., Groma, I., Györgyi, G., Csikor, F.F., Weygand, D., 2010. Submicron Plasticity: Yield Stress, Dislocation Avalanches, and Velocity Distribution. Phys. Rev. Lett. 105.

Kelley, E.W., Hosford, W.F., 1968. Plane-Strain Compression of Magnesium and Magnesium Alloy Crystals. T. Metall. Soc. Aime 242, 5-11.

Khan, A.S., Pandey, A., Gnaupel-Herold, T., Mishra, R.K., 2011. Mechanical response and texture evolution of AZ31 alloy at large strains for different strain rates and temperatures. Int. J. Plast. 27, 688-706.

Kontsos, A., Loutas, T., Kostopoulos, V., Hazeli, K., Anasori, B., Barsoum, M.W., 2011. Nanocrystalline Mg-MAX composites: Mechanical behavior characterization via acoustic emission monitoring. Acta Mater. 59, 5716-5727.

Lebensohn, R.A., Tomé, C.N., 1993. A Self-Consistent Anisotropic Approach for the Simulation of Plastic-Deformation and Texture Development of Polycrystals - Application to Zirconium Alloys. Acta Metall Mater 41, 2611-2624.

Lebyodkin, M.A., Shashkov, I.V., Lebedkina, T.A., Máthis, K., Dobroň, P., Chmelík, F., 2013. Role of superposition of dislocation avalanches in the statistics of acoustic emission during plastic deformation. Phys. Rev. E 88 Art. Nr. 042402.

Levesque, J., Inal, K., Neale, K.W., Mishra, R.K., 2010. Numerical modeling of formability of extruded magnesium alloy tubes. Int. J. Plast. 26, 65-83.

Lou, X.Y., Li, M., Boger, R.K., Agnew, S.R., Wagoner, R.H., 2007. Hardening evolution of AZ31B Mg sheet. Int. J. Plast. 23, 44-86.

Lu, Y., Gharghouri, M., Taheri, F., 2008. Effect of texture on acoustic emission produced by slip and twinning in AZ31B magnesium alloy - part II: clustering and neural network analysis. NDT Eval. 23, 211-228.

Máthis, K., Čapek, J., Zdražilová, Z., Trojanová, Z., 2011. Investigation of tension-compression asymmetry of magnesium by use of the acoustic emission technique. Mater. Sci. Eng. A 528, 5904-5907.

Máthis, K., Chmelík, F., Janeček, M., Hadzima, B., Trojanová, Z., Lukáč, P., 2006. Investigating deformation processes in AM60 magnesium alloy using the acoustic emission technique. Acta Mater. 54, 5361-5366.

Máthis, K., Nyilas, K., Axt, A., Dragomir-Cernatescu, I., Ungár, T., Lukáč, P., 2004. The evolution of non-basal dislocations as a function of deformation temperature in pure magnesium determined by X-ray diffraction. Acta Mater. 52, 2889-2894.

Mayama, T., Noda, M., Chiba, R., Kuroda, M., 2011. Crystal plasticity analysis of texture development in magnesium alloy during extrusion. Int J Plast. 27, 1916-1935. 
Mecking, H., Kocks, U.F., 1981. Kinetics of Flow and Strain-Hardening. Acta Metall. Mater. 29, 1865-1875.

Mendelson, S., 1970. Dislocation Dissociations in Hcp Metals. J Appl. Phys. 41, 1893-1911.

Mordike, B.L., Ebert, T., 2001. Magnesium - Properties - applications - potential. Mater. Sci. Eng. A 302, 37-45.

$\mathrm{Mu}$, S., Jonas, J.J., Gottstein, G., 2012. Variant selection of primary, secondary and tertiary twins in a deformed Mg alloy. Acta Mater. 60, 2043-2053.

Muránsky, O., Barnett, M.R., Carr, D.G., Vogel, S.C., Oliver, E.C., 2010a. Investigation of deformation twinning in a fine-grained and coarse-grained ZM20 Mg alloy: Combined in situ neutron diffraction and acoustic emission. Acta Mater. 58, 1503-1517.

Muránsky, O., Barnett, M.R., Luzin, V., Vogel, S., 2010b. On the correlation between deformation twinning and Luders-like deformation in an extruded $\mathrm{Mg}$ alloy: In situ neutron diffraction and EPSC.4 modelling. Mater. Sci. Eng. A 527, 1383-1394.

Neil, C.J., Wollmershauser, J.A., Clausen, B., Tomé, C.N., Agnew, S.R., 2010. Modeling lattice strain evolution at finite strains and experimental verification for copper and stainless steel using in situ neutron diffraction. Int. J. Plast. 26, 1772-1791.

Niewczas, M., 2007. Dislocations and twinning in face centered cubic crystals. Elsevier Science. Oppedal, A.L., El Kadiri, H., Tomé, C.N., Kaschner, G.C., Vogel, S.C., Baird, J.C., Horstemeyer, M.F., 2012. Effect of dislocation transmutation on modeling hardening mechanisms by twinning in magnesium. Int. J. Plast. 30-31, 41-61.

Papirov, I.I., Karpov, E.S., Palatnik, M.I., Mileshkin, M.B., 1984. Acoustic-Emission during Plastic and Superplastic Deformation of a Zn-0.4-percent Al-Alloy. Met. Sci. Heat. Treat+ 26, 887-891.

Pomponi, E., Vinogradov, A., 2013. A real-time approach to acoustic emission clustering. Mech. Syst. Signal Pr. 40, 791-804.

Proust, G., Tomé C.N., Jain, A., Agnew, S.R., 2009. Modeling the effect of twinning and detwinning during strain-path changes of magnesium alloy AZ31. Int J Plast. 25, 861-880.

Scruby, C., Wadley, H., Sinclair, J.E., 1981. The oirigin of acoustic-emission during deformation of aluminum and an aluminum-magnesium alloy. Philos.. Mag. A 44, 249-274.

Serra, A., Bacon, D.J., 2010. Interaction of a moving $\{10-12\}$ twin boundary with perfect dislocations and loops in a hcp metal. Philos Mag. 90, 845-861.

Serra, A., Bacon, D.J., Pond, R.C., 1999. Dislocations in interfaces in the hcp metals - I. Defects formed by absorption of crystal dislocations. Acta Mater. 47, 1425-1439.

Serra, A., Pond, R.C., Bacon, D.J., 1991. Computer-Simulation of the Structure and Mobility of Twinning Dislocations in Hcp Metals. Acta Metall. Mater. 39, 1469-1480.

Sibil, A., Godin, N., R'Mili, M., Maillet, E., Fantozzi, G., 2012. Optimization of Acoustic Emission Data Clustering by a Genetic Algorithm Method. J. Nondestruct. Eval. 31, 169-180.

Stanford, N., Barnett, M.R., 2013. Solute strengthening of prismatic slip, basal slip and $\left\{\begin{array}{lll}1 & 0 & -12\end{array}\right\}$ twinning in $\mathrm{Mg}$ and $\mathrm{Mg}$-Zn binary alloys. Int. J. Plast. 47, 165-181.

Staroselsky, A., Anand, L., 2003. A constitutive model for hep materials deforming by slip and twinning: application to magnesium alloy AZ31B. Int J Plast. 19, 1843-1864.

Styczynski, A., Hartig, C., Bohlen, J., Letzig, D., 2004. Cold rolling textures in AZ31 wrought magnesium alloy. Scripta Mater. 50, 943-947.

Tomé, C.N., Beyerlein, I.J., Wang, J., McCabe, R.J., 2011. A multi-scale statistical study of twinning in magnesium. Jom-Us 63, 19-23.

Turner, P.A., Tomé, C.N., Woo, C.H., 1994. Self-Consistent Modeling of Nonlinear Viscoelastic Polycrystals - an Approximate Scheme. Philos. Mag. A 70, 689-711. 
Ungár, T., 2004. Micro structural parameters from X-ray diffraction peak broadening. Scripta Mater. 51, 777-781.

Vinogradov, A., Lazarev, A., 2012. Continuous acoustic emission during intermittent plastic flow in $\alpha$-brass. Scripta Mater. 66, 745-748.

Vinogradov, A., Lazarev, A., Linderov, M., Weidner, A., Biermann, H., 2013a. Kinetics of deformation processes in high-alloyed cast transformation-induced plasticity/twinning-induced plasticity steels determined by acoustic emission and scanning electron microscopy: Influence of austenite stability on deformation mechanisms. Acta Mater. 61, 2434-2449.

Vinogradov, A., Orlov, D., Danyuk, A., Estrin, Y., 2013b. Effect of grain size on the mechanisms of plastic deformation in wrought $\mathrm{Mg}-\mathrm{Zn}-\mathrm{Zr}$ alloy revealed by acoustic emission measurements. Acta Mater. 61, 2044-2056.

Vinogradov, A., Yasnikov, I.S., Estrin, Y., 2014. Stochastic dislocation kinetics and fractal structures in deforming metals probed by acoustic emission and surface topography measurements. J .Appl. Phys. 115.

Wang, H., Wu, P.D., Wang, J., 2013a. Modeling inelastic behavior of magnesium alloys during cyclic loading-unloading. Int J Plasticity 47, 49-64.

Wang, H., Wu, P.D., Wang, J., Tomé, C.N., 2013b. A crystal plasticity model for hexagonal close packed (HCP) crystals including twinning and de-twinning mechanisms. Int. J. Plast. 49, 36-52.

Wen, W., Borodachenkova, M., Tomé, C.N., Vincze, G., Rauch, E.F., Barlat, F., Grácio, J.J., 2014. Mechanical behavior of Mg subjected to strain path changes: Experiments and modeling. Int. J. Plast. in press, http://dx.doi.org-10.1016/j.ijplas.2014.20.009

Wilkens, M., 1969. Theoretical Aspects of Kinematical X-Ray Diffraction Profiles from Crystals Containing Dislocation Distributions. J. Res. Nbs. a Phys. Ch. A 73, 552-557.

$\mathrm{Xu}, \mathrm{B}$., Capolungo, L., Rodney, D., 2013. On the importance of prismatic/basal interfaces in the growth of (1012) twins in hexagonal close packed crystals. Scripta Mater. 68, 901-904. Yoshinaga, H., Horiuchi, R., 1964. On nonbasal slip in magnesium crystals. Trans. JIM 5, 14-21. Yu, Q., Wang, J., Jiang, Y.Y., McCabe, R.J., Li, N., Tomé, C.N., 2014. Twin-twin interactions in magnesium. Acta Mater. 77, 28-42. 


\section{Figure captions}

Fig. 1 - Inverse pole figures for the initial state of the specimens as measured by neutron diffraction in the axial detector (Bank2) a) tension; b) compression

Fig. 2 - AE waveform stream and the corresponding monotonic stress - strain curves a) in tension; b) in compression.

Fig. 3 - The shapes of the power spectral density (PSD) functions corresponding to the particular clusters identified using the ASK algorithm for tensile AE data.

Fig. 4 - Distribution of AE clusters in energy - mean frequency space including the 95\% confidence ellipse for tensile AE data. The clusters, represented by different colors, are assigned to particular source mechanisms, based on their characteristic features (energy, frequency distribution etc.). The distribution of the clusters in compression looks similar, therefore is not presented here.

Fig. 5 - Distribution of AE clusters in mean frequency - kurtosis - energy 3D space. The $z$-axis represents number of $\mathrm{AE}$ events, which have their energy:

a) $\boldsymbol{E}<5 \times 10^{-4}$ a.u.;

b) $5 \times 10^{-4}$ a.u. $<\boldsymbol{E}<5 \times 10^{-3}$ a.u.;

c) $\boldsymbol{E}>5 \times 10^{-3}$ a.u.;

The color code used for clusters is the same as in Fig. 4.

Fig. 6 - Strain evolution of cumulative number of elements in the AE clusters assigned to a) noise (grey line), basal dislocations (red line) and non-basal dislocations (orange line);

b) twinning 1 (blue line) and twinning 2 (magenta line).

The black line represents the experimental stress strain curves, measured concurrently with AE. In the left upper corner of figures the characteristic waveforms of dislocations slip (red colored Fig. 6a) and twinning (blue colored - Fig. 6b) are shown.

Fig. 7 - Strain evolution of cumulative energy of the AE events in the particular clusters a) in tension; b) in compression.

Fig. 8: CMWP fitting for the the initial sample and sample deformed by compression up to $6 \%$. The pattern was obtained by the radial detector. The open circles and the solid line represent the measured data and the fitted curves, respectively. The intensity is plotted in logarithmic scale. The inset shows a part of the diffractogram with larger magnification. In the inset the intensity is plotted in linear scale and the difference between the measured and the fitted patterns is shown at the bottom.

Fig. 9 - Classical (a) and modified (b) Williamson-Hall plots for the sample deformed by compression up to the strain of $6 \%$. The profiles were measured by the radial detector and corrected for the instrumental effects. FWHM: full width at half maximum, $g$ : length of the diffraction vector, $\mathrm{C}$ : dislocation contrast factor.

Fig. 10 - The dislocation density as a function strain for tension and compression. 
Fig. 11 - The ratios of $\langle a\rangle\langle\langle c+a\rangle$ and basal/prismatic dislocations ( $\langle a\rangle$-types) versus strain for tensile deformed (a,b) and compressed (c,d) samples as detected by radial and axial detectors.

Notaition used: BE: basal edge, PrE: prismatic edge.

Fig. 12 - The experimental and calculated (using EPSC model) stress-strain curves for both loading modes.

Fig. 13 - The calculated (left column) and measured (right column - axial detector) inverse pole figures at the strains of 0,3 and $6 \%$ for tension (a) and compression (b)

Fig. 14 - The comparison of twinned volume in tension (red color) and compression (black color), estimated by EPSC model (line) and measured (symbols) using neutron diffraction (the experimental data are taken from (Čapek et al., 2014)).

Fig. 15 - Calculated numbers of nucleated twin variants for 20000 grains and twin variants nucleated per grain for tension and compression.

Fig. 16 - EBSD orientation maps of specimens deformed up to $1 \%$ of strain; a) in compression; b) and c) in tension.

Fig. 17 - Activity of particular deformation mechanisms as a function of the applied stress calculated using EPSC model: overall (left); in parent grains (middle); in twins (right)

(a) in tension

(b) in compression.

Fig. 18 - Experimental (symbols) and calculated (lines) internal strain evolution versus the applied stress both in tension and compression for both detector banks.

Fig. 19 - Comparison of relative activity of main deformation processes (basal and non-basal slip, twinning) for both loading modes (a) - tension; (b) - compression; versus the applied stress obtained by EPSC modeling and estimated from AE measurements. 
Figure 01

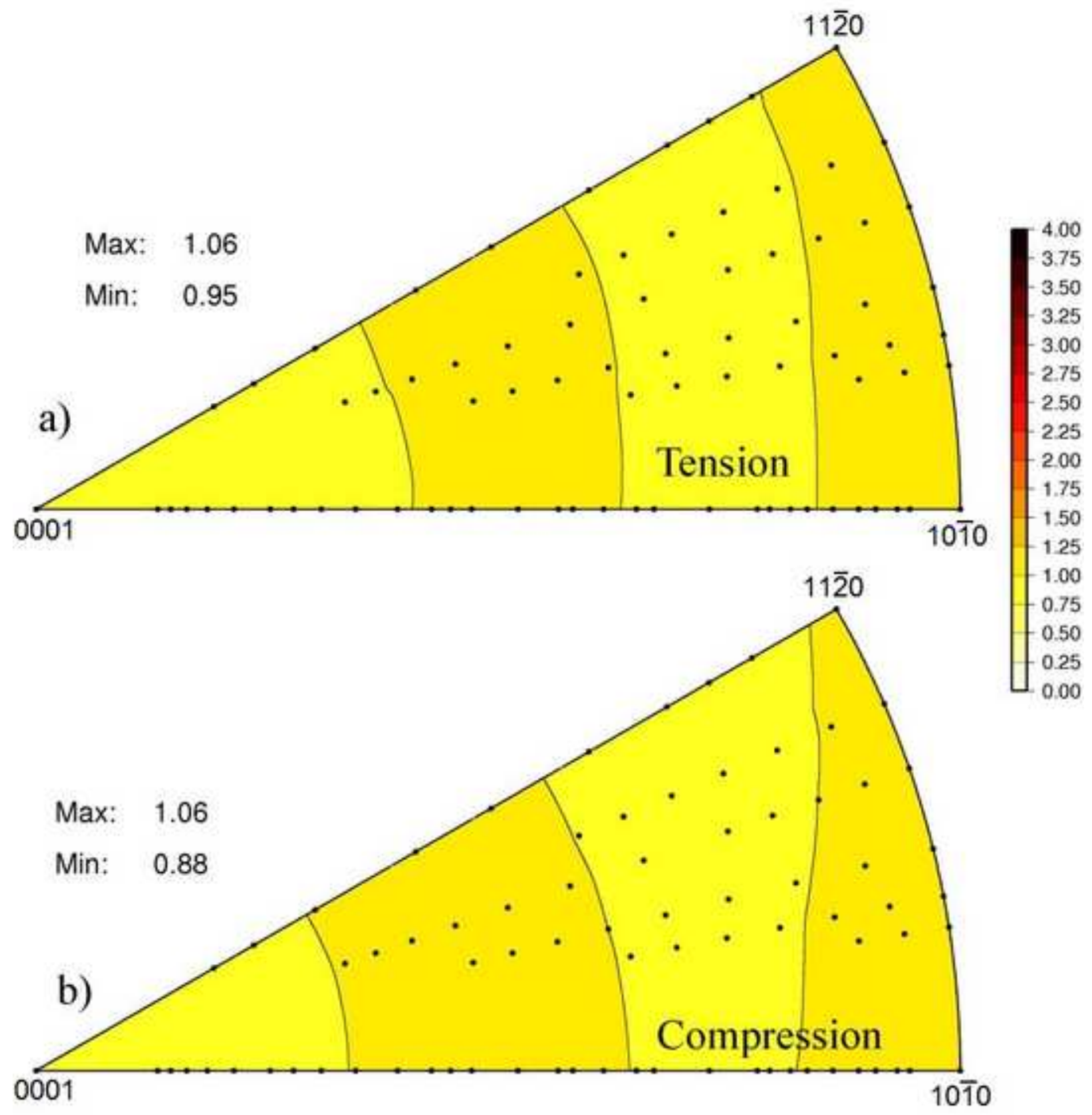




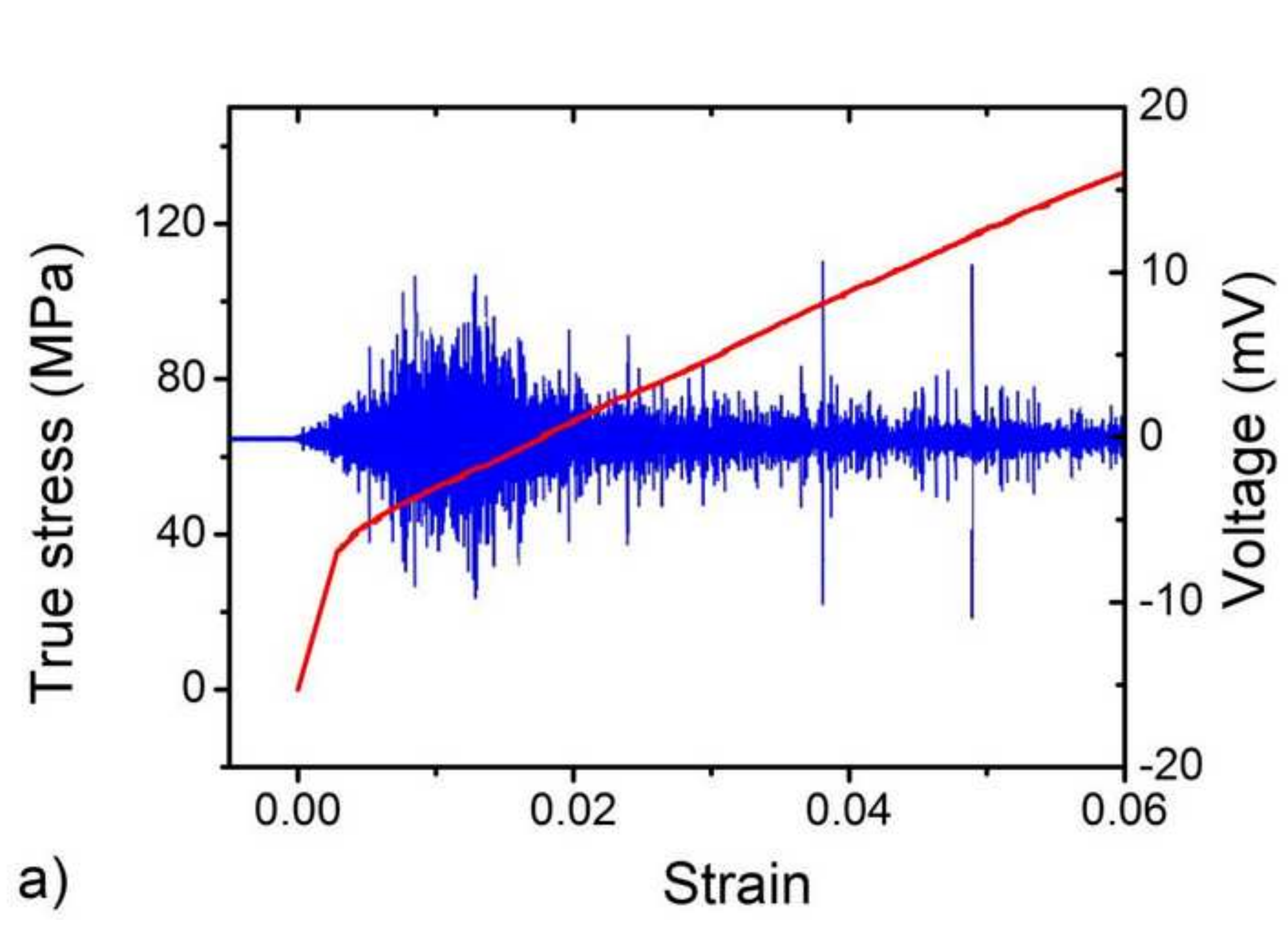




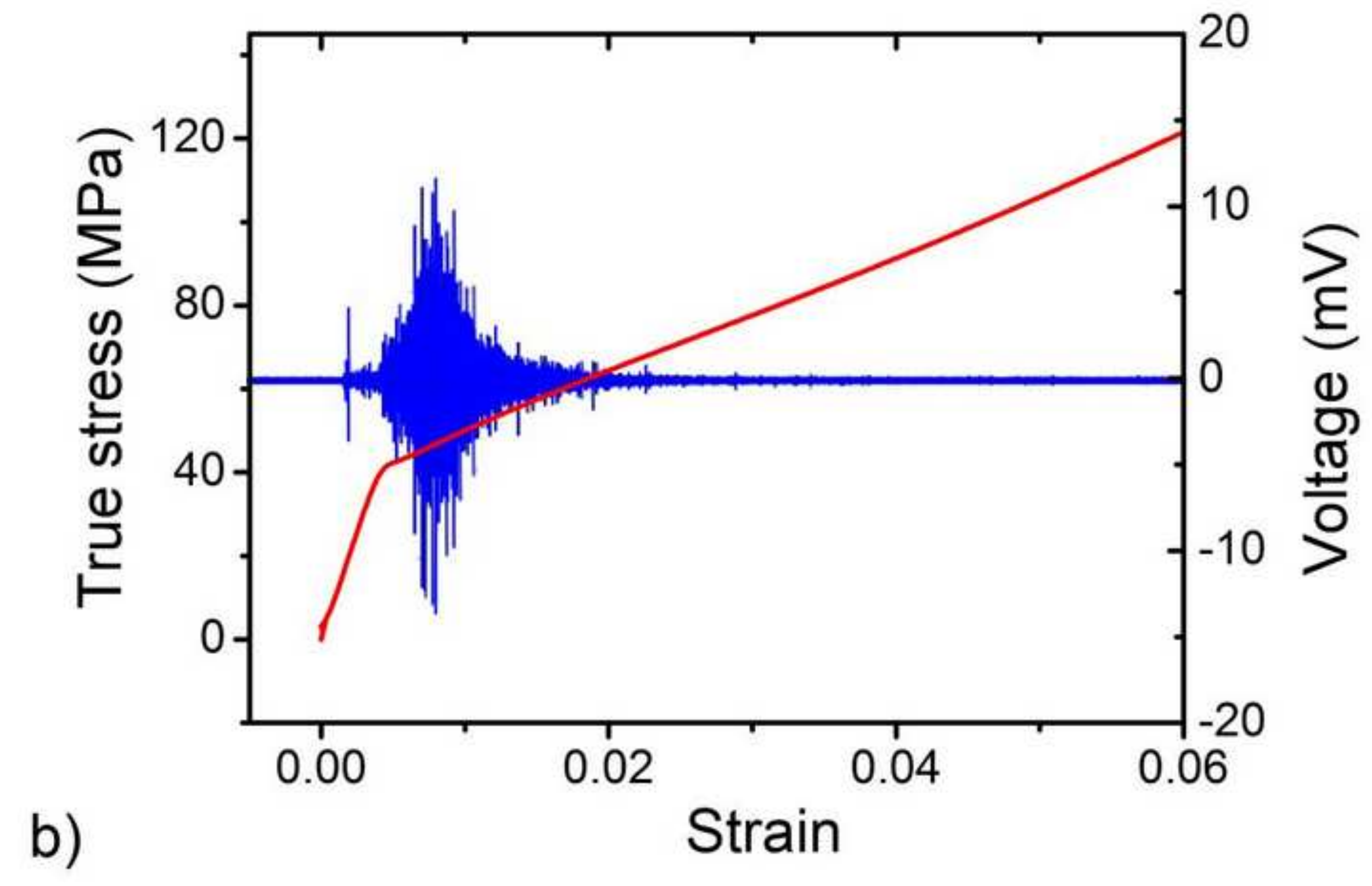

b)

Strain 


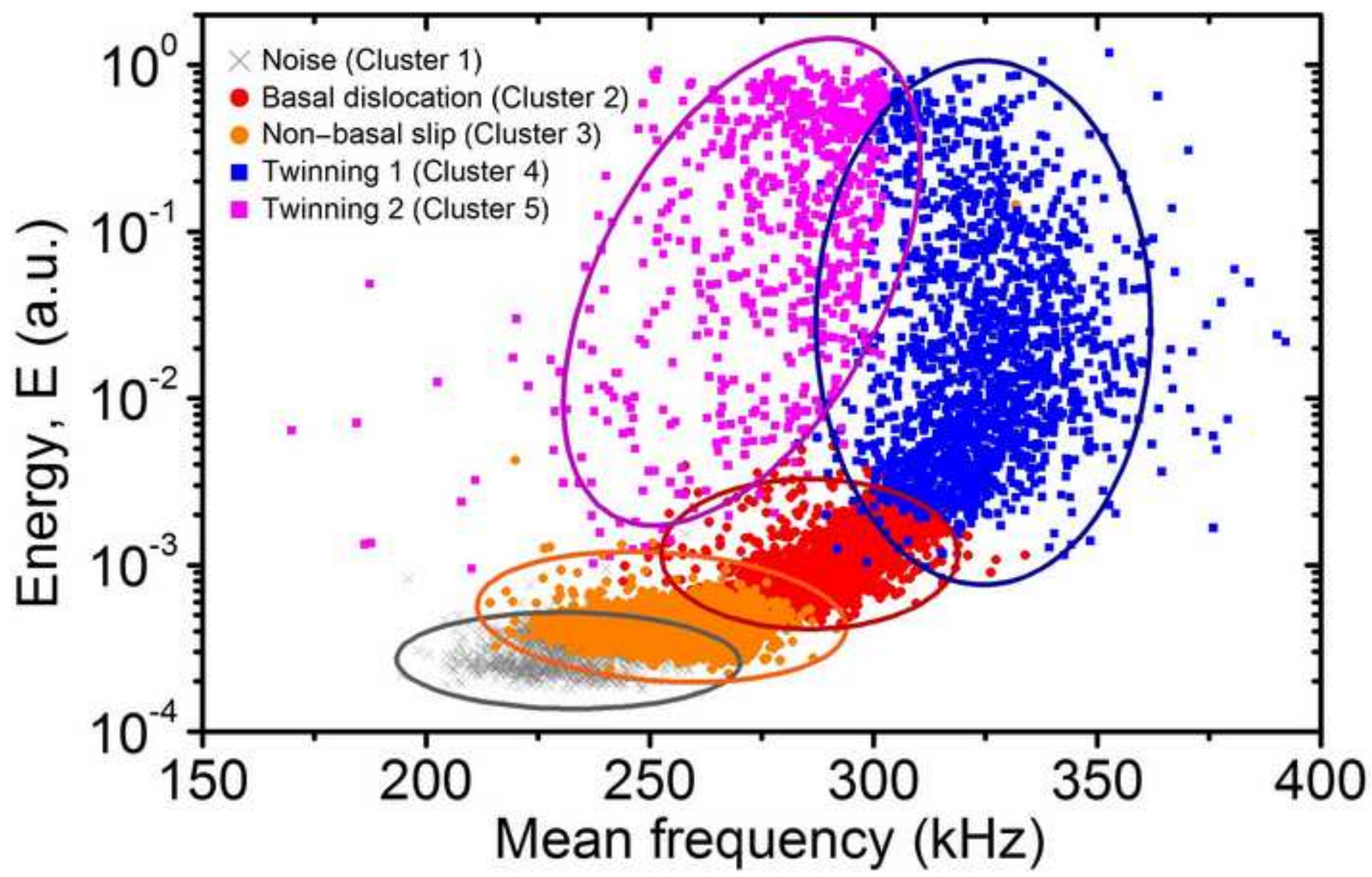




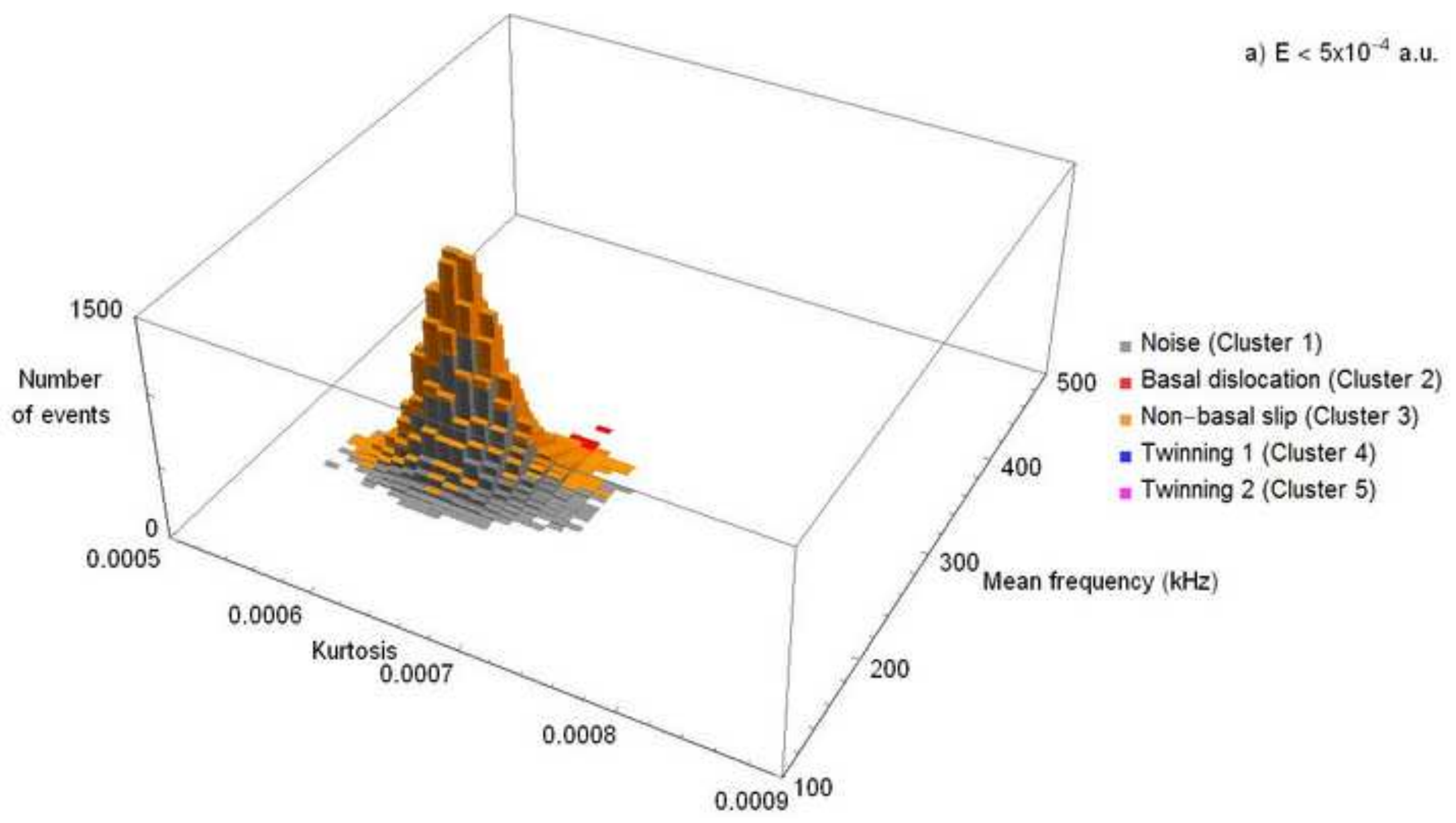




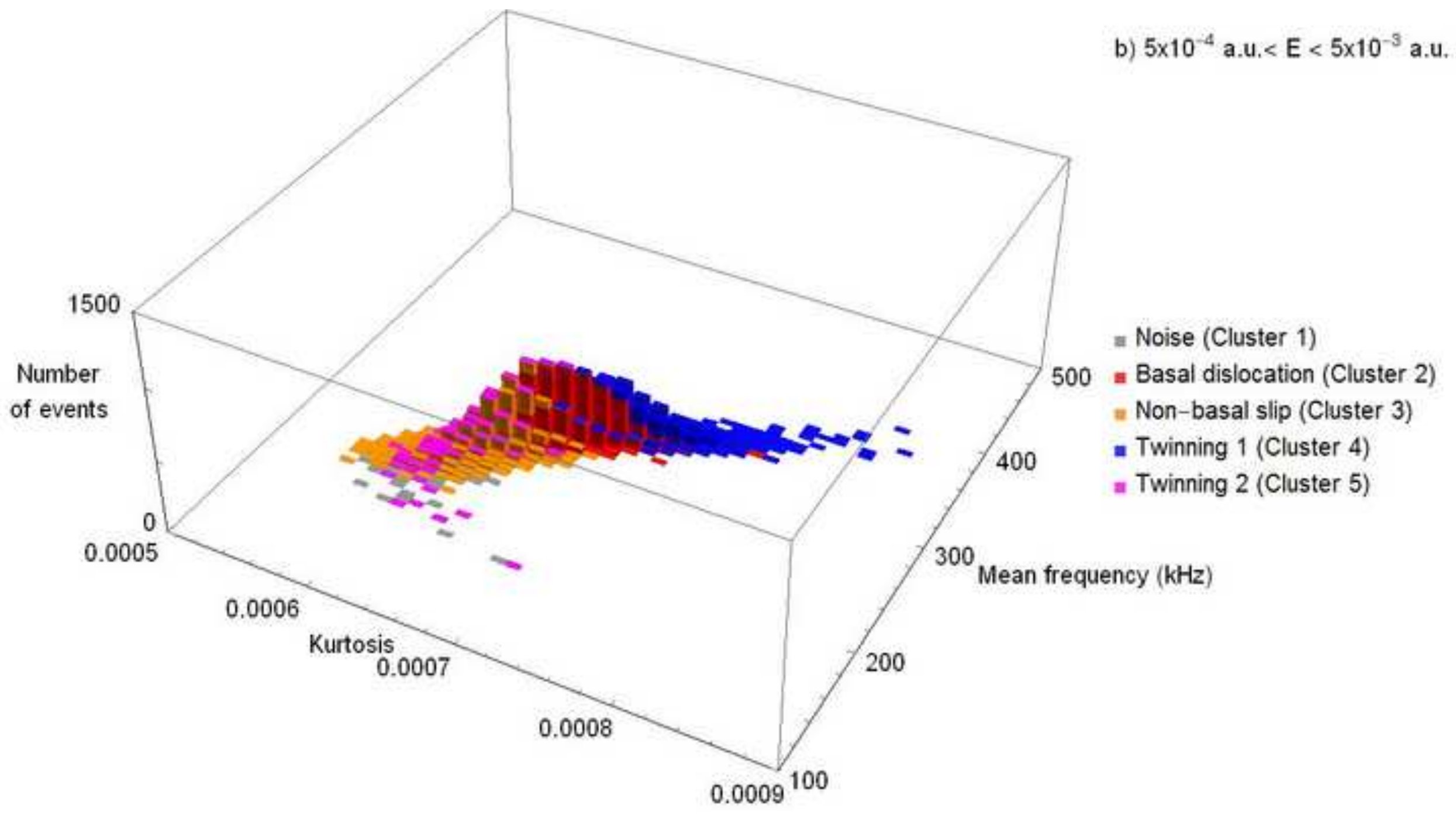




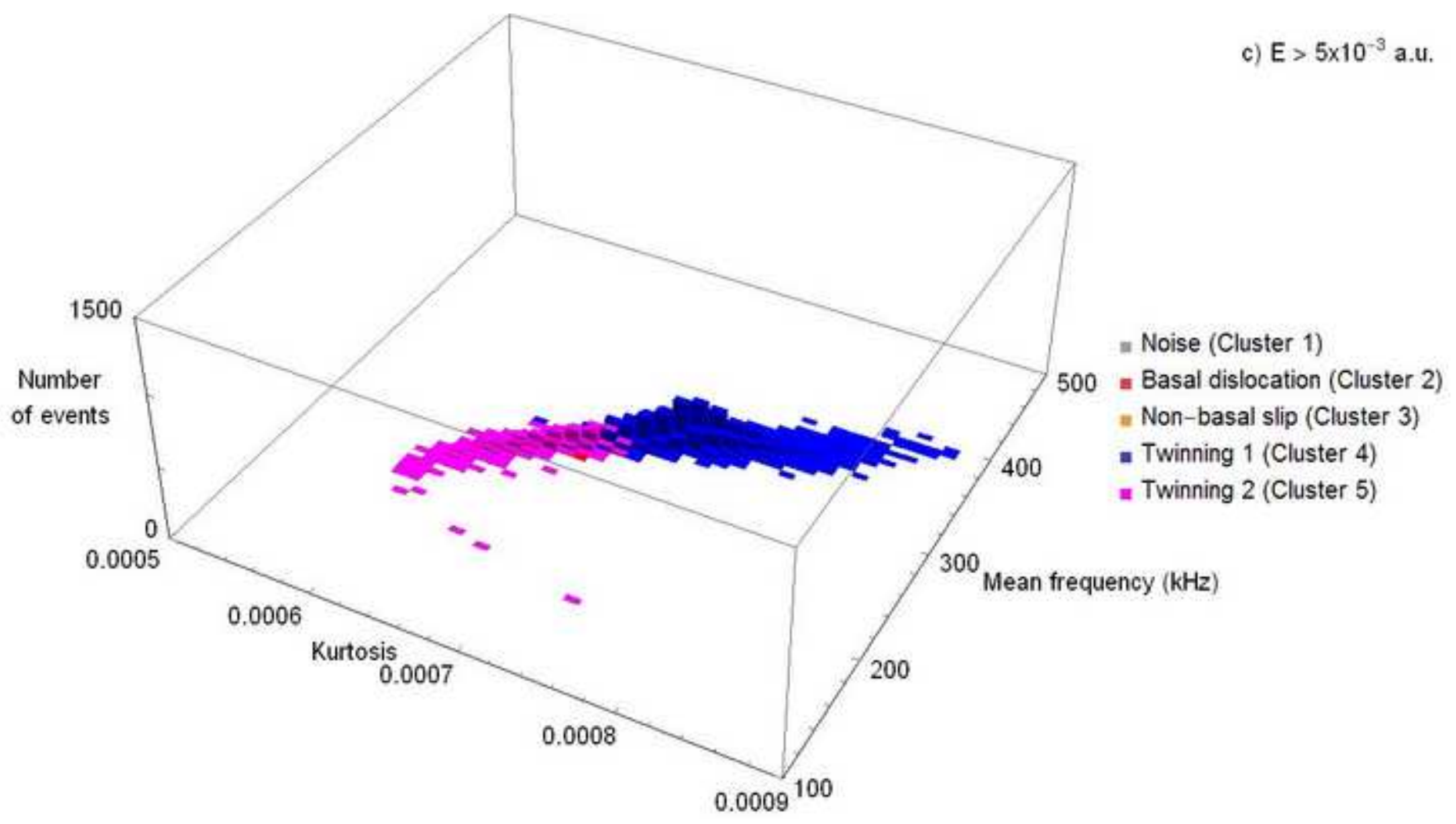




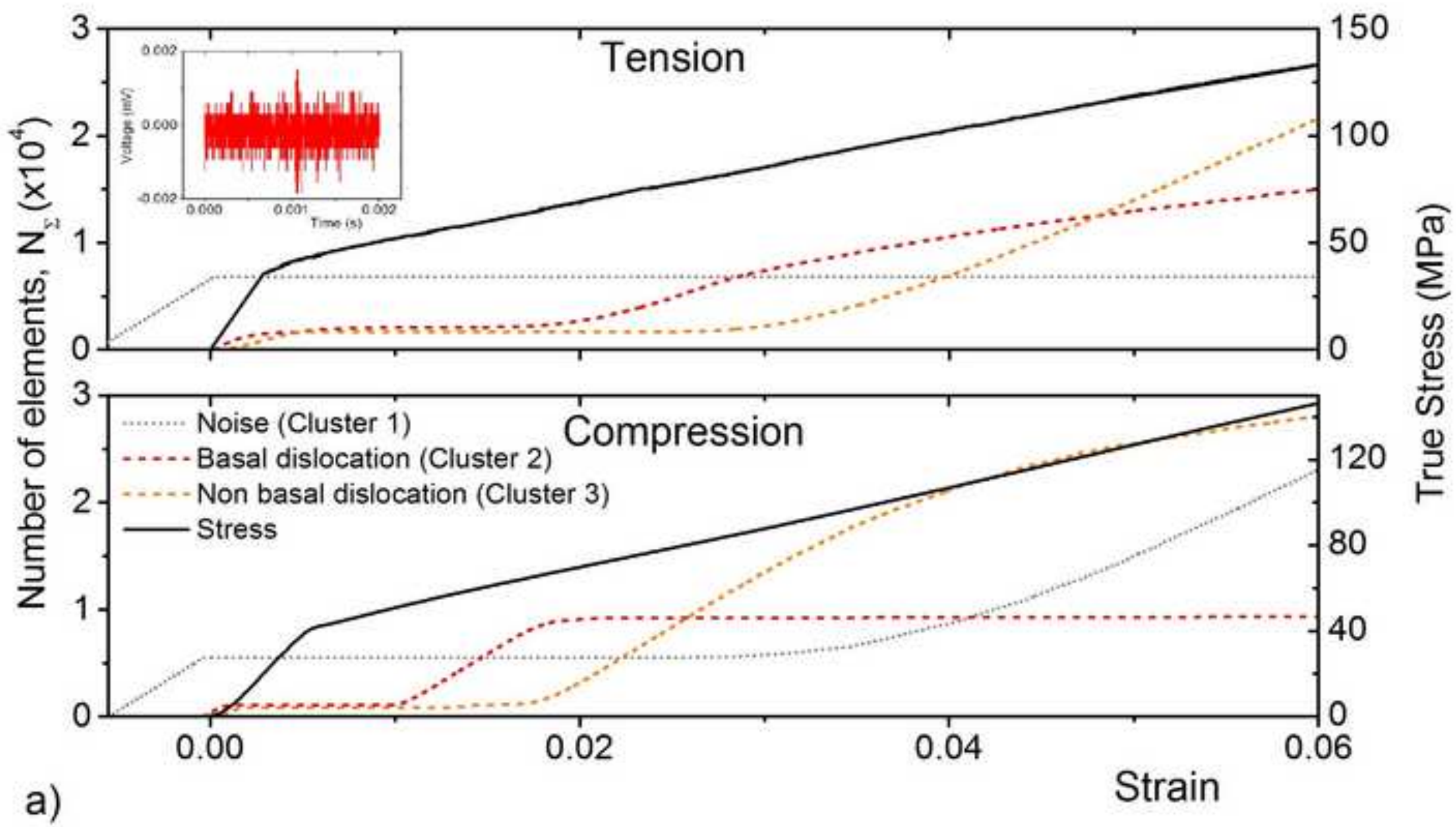

a)

?

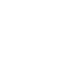




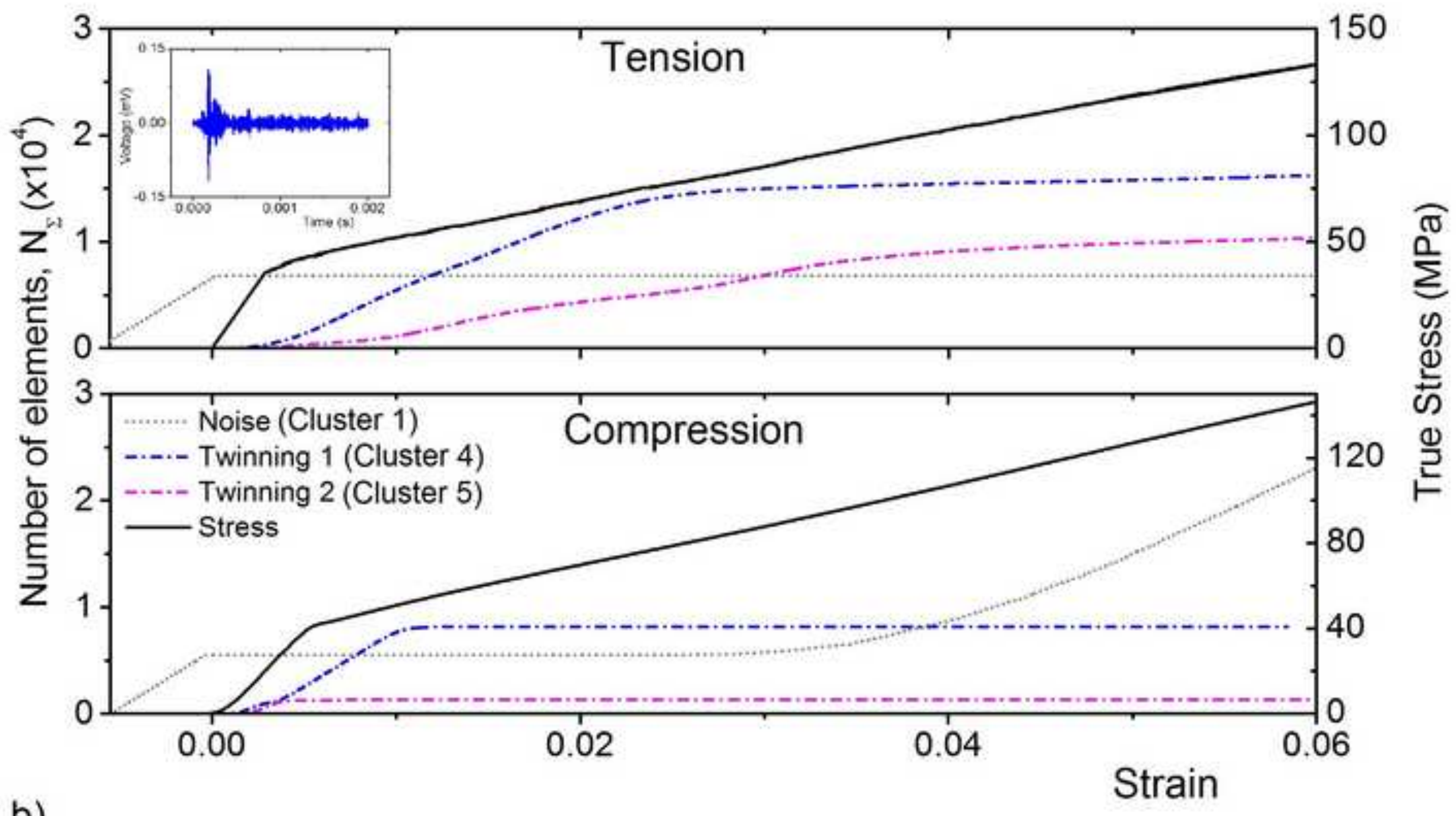

b) 


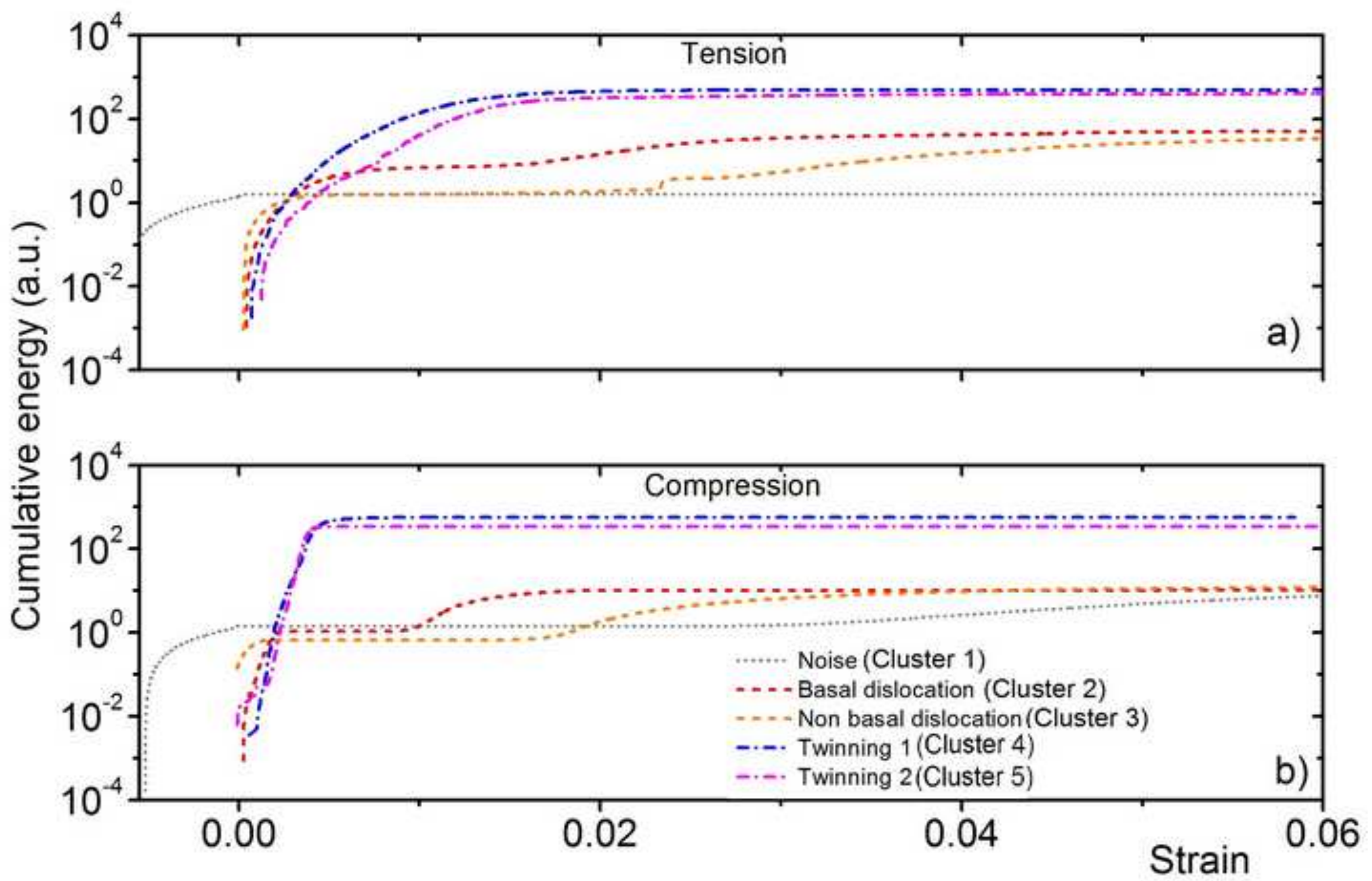




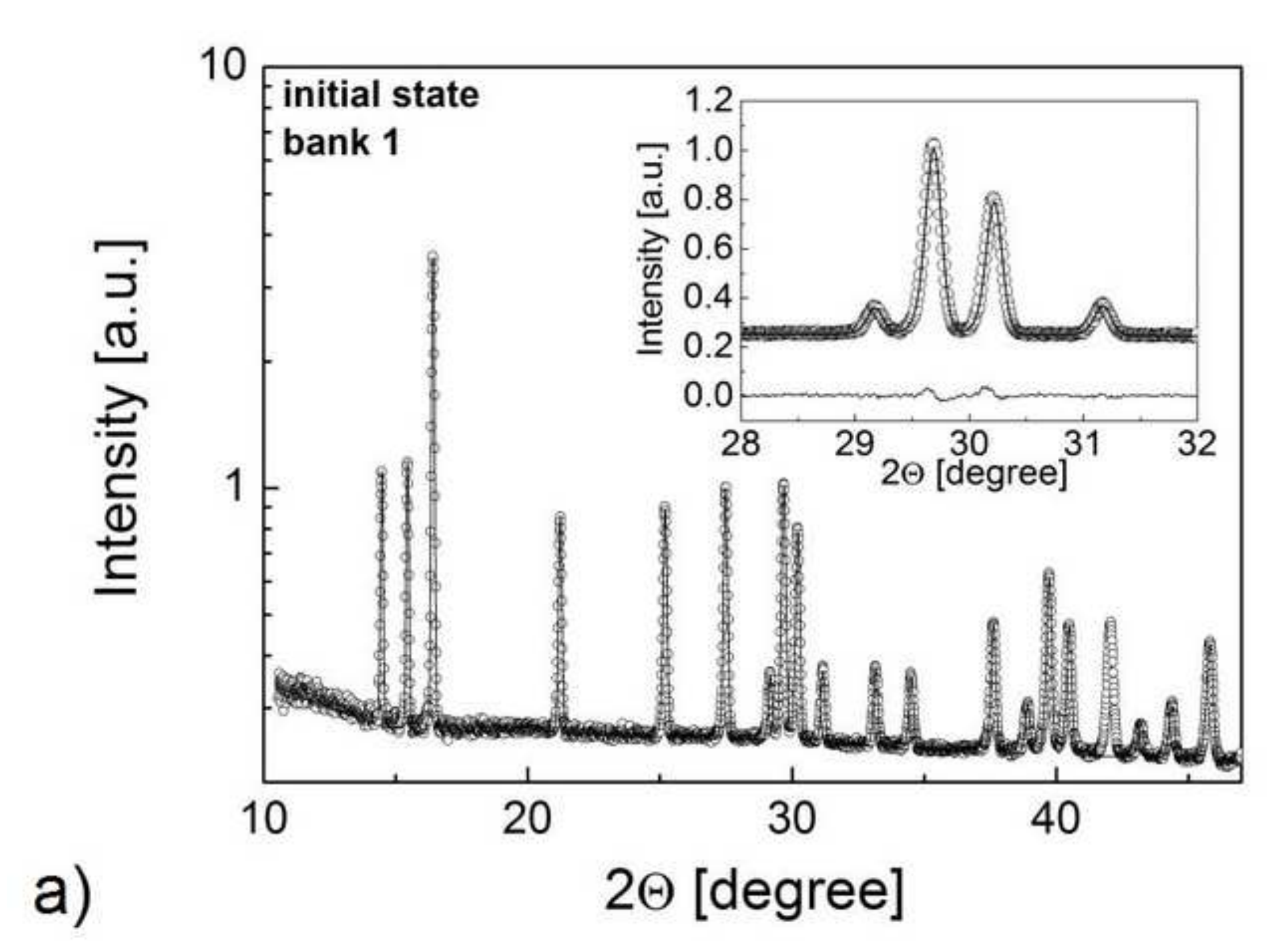




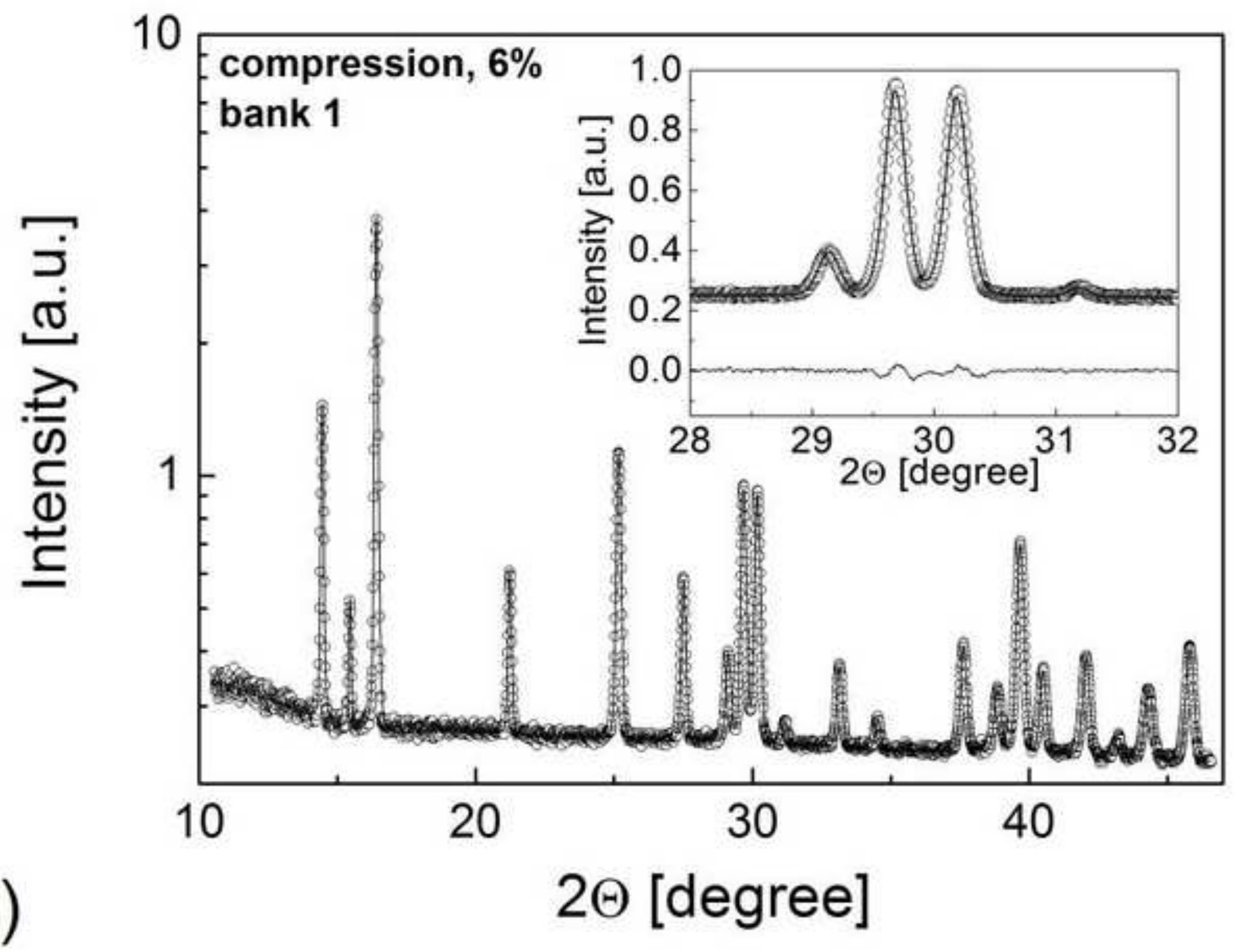

b)

$2 \Theta$ [degree] 


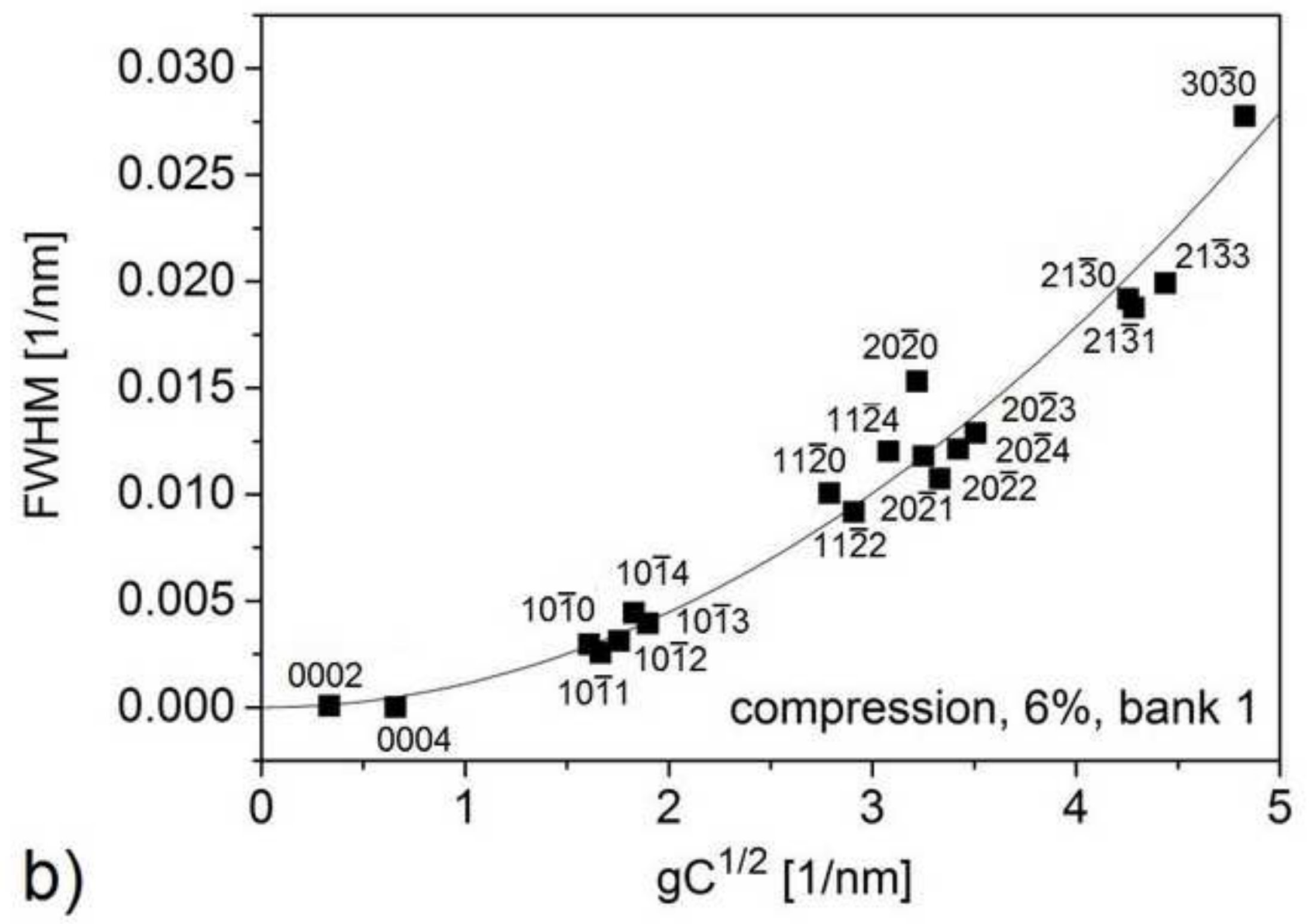




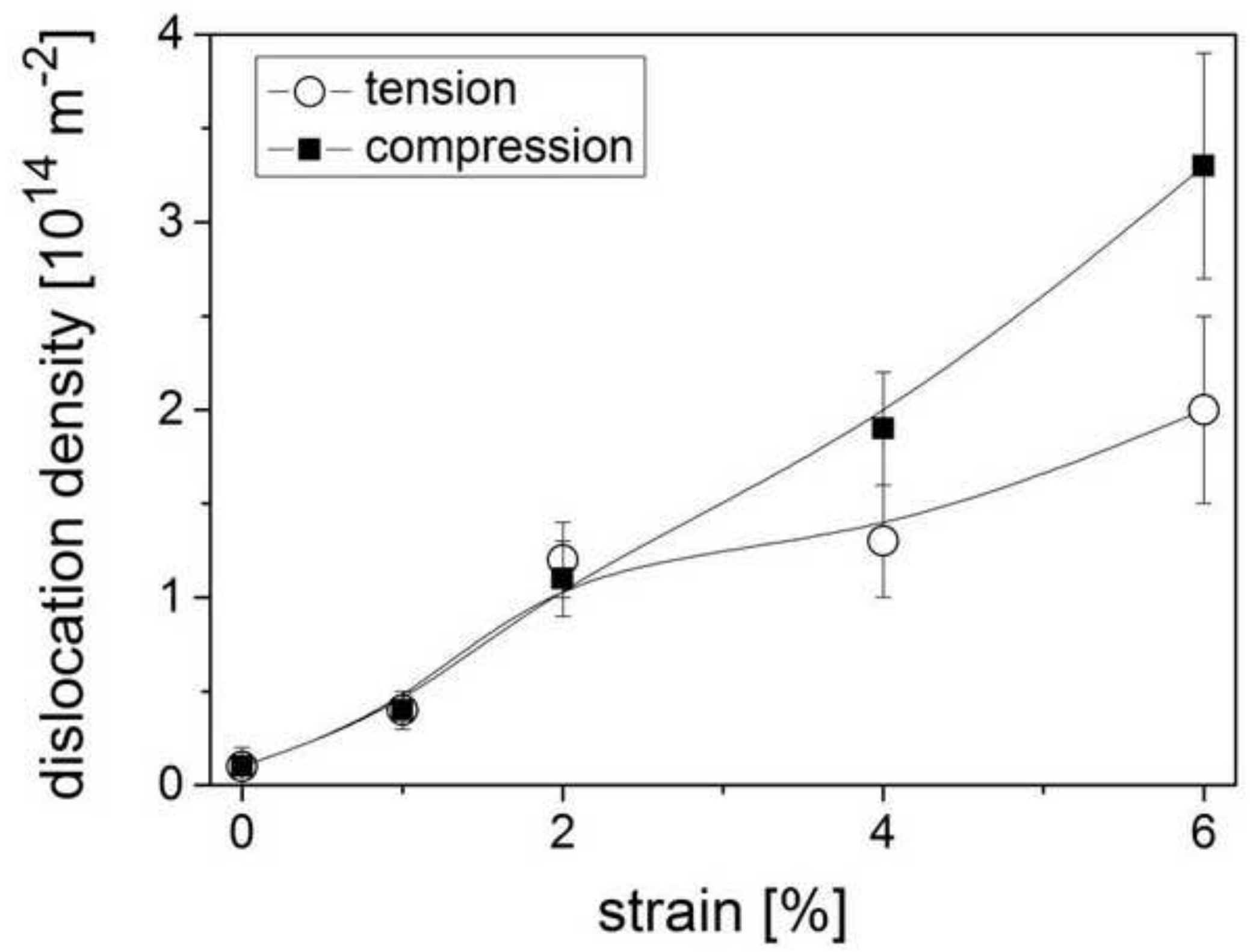




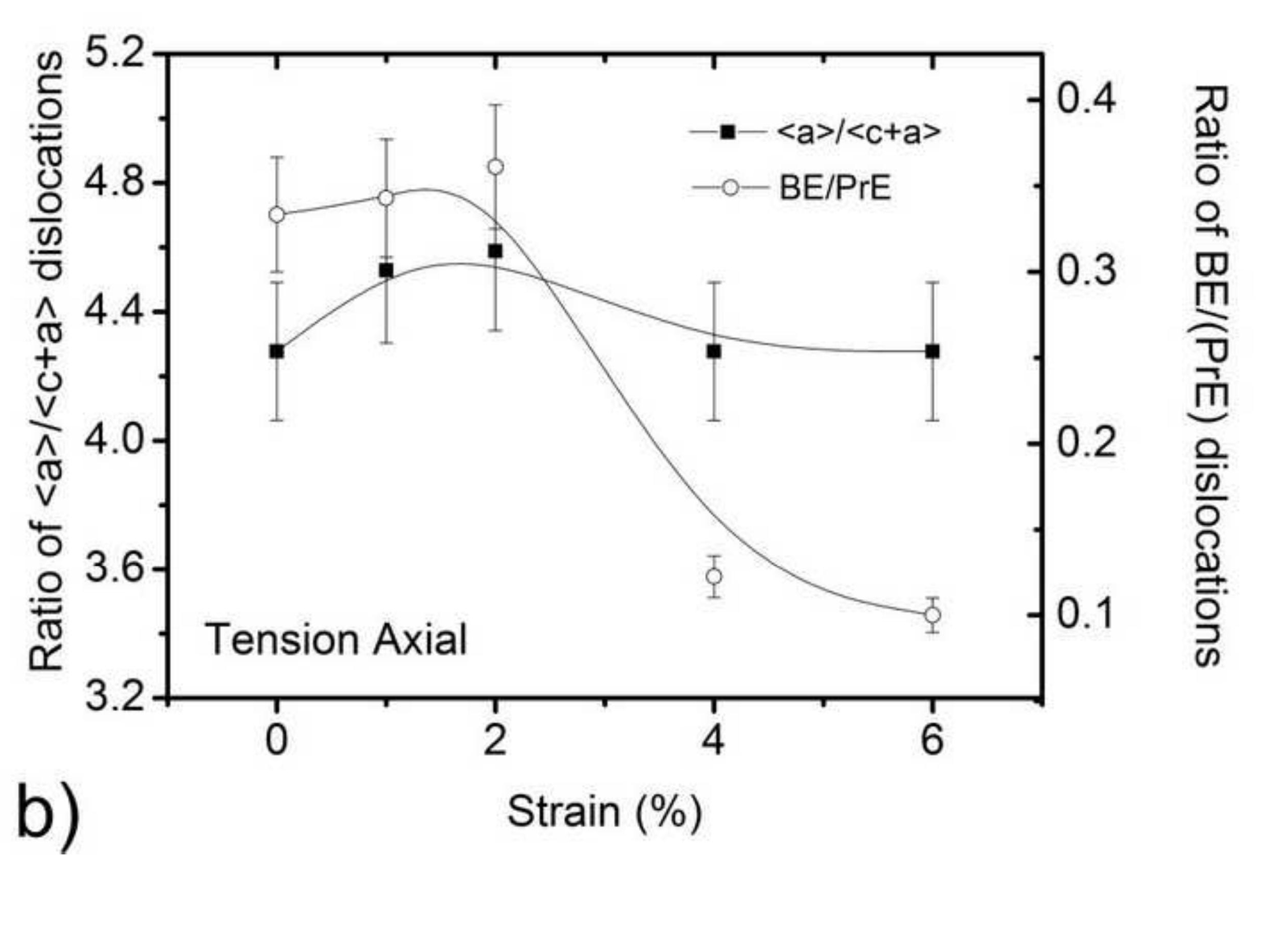




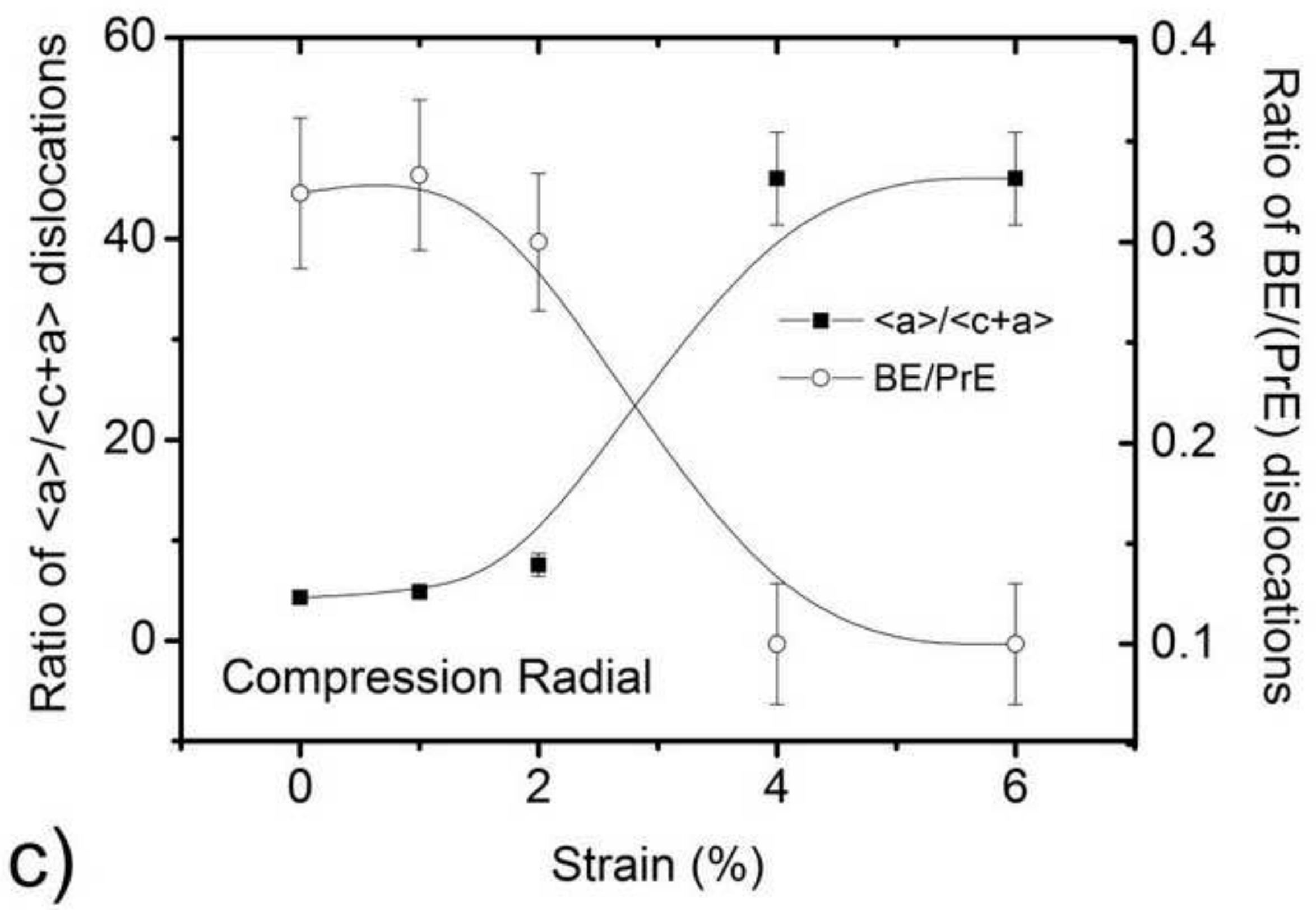




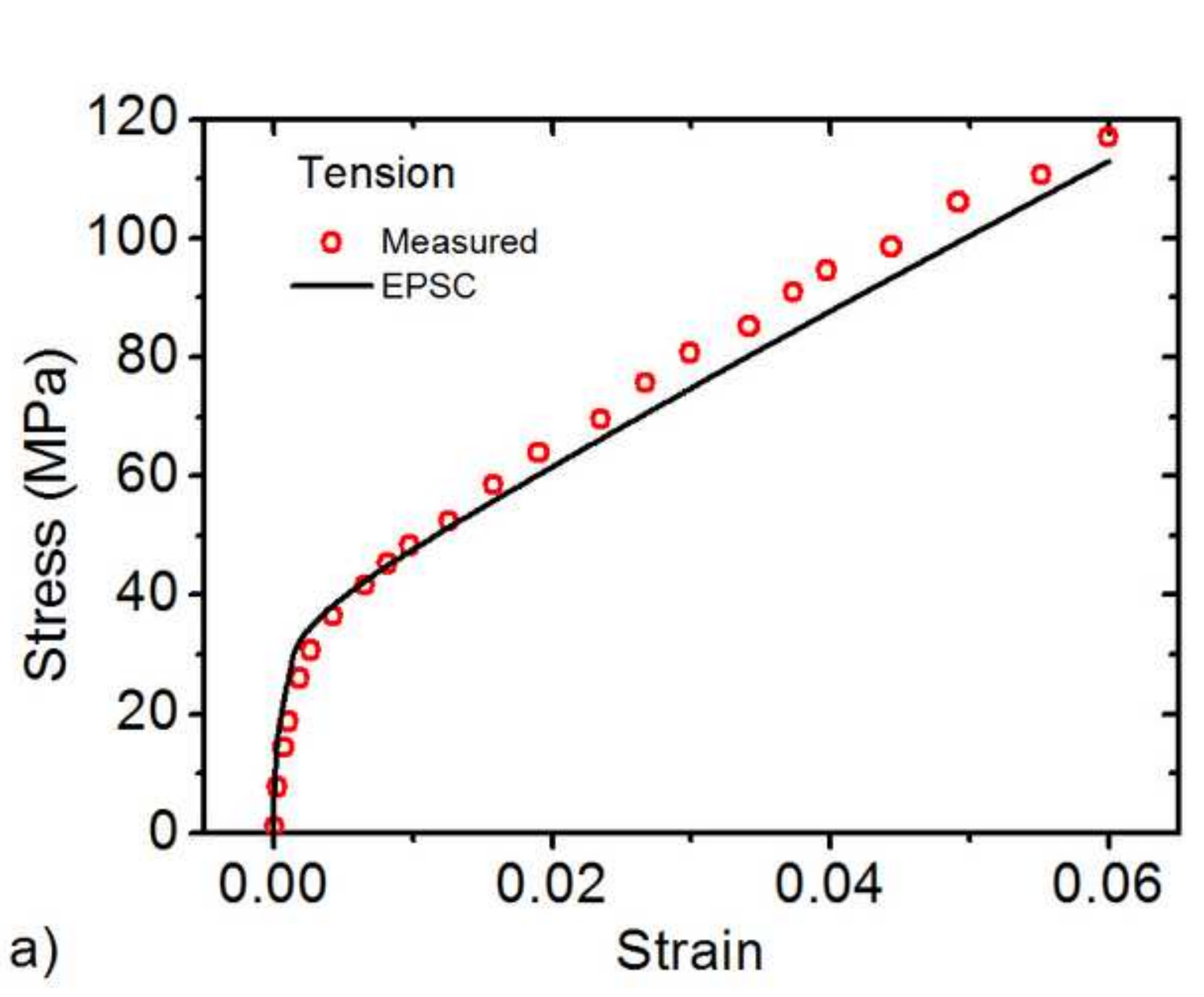




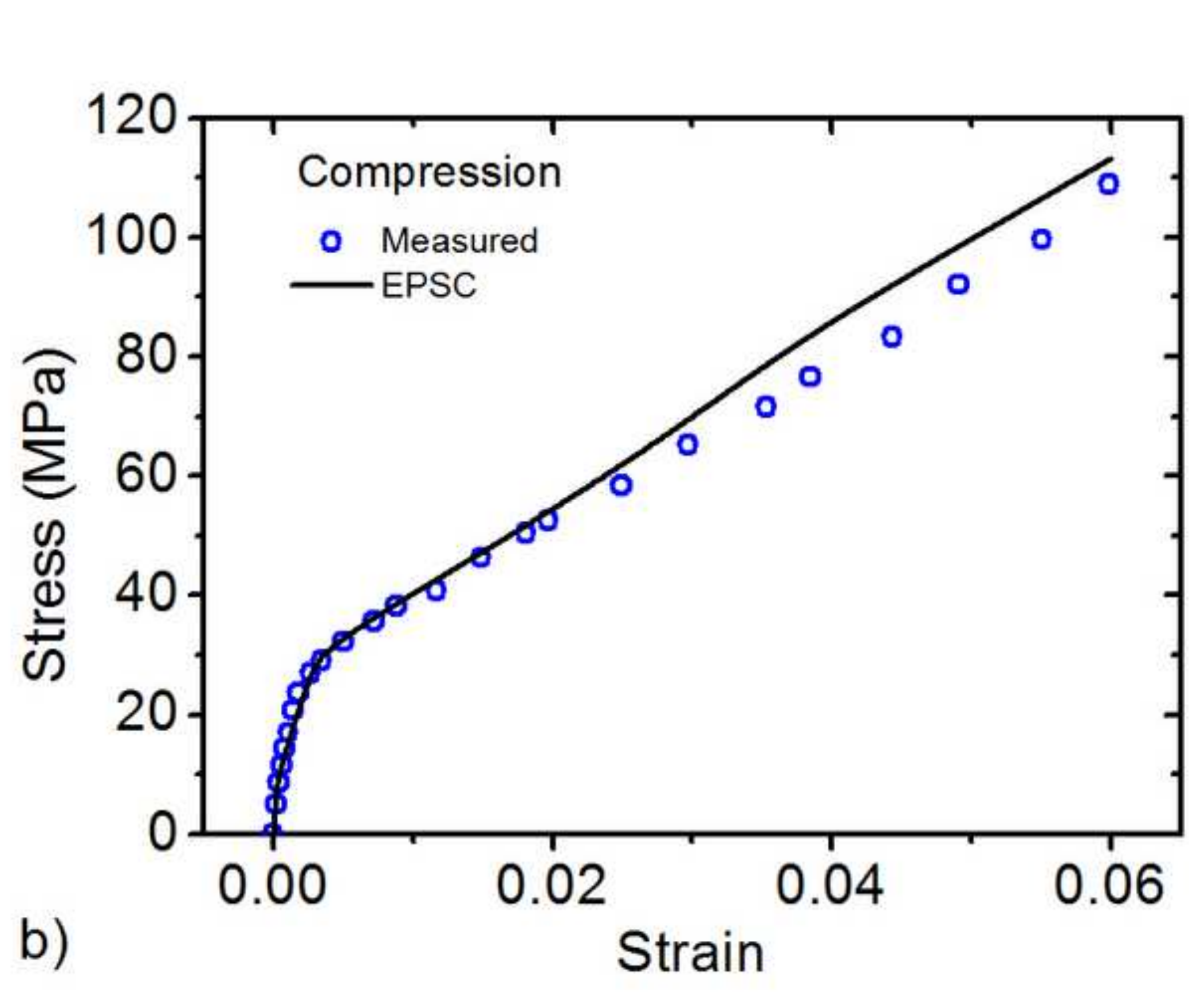


Compression
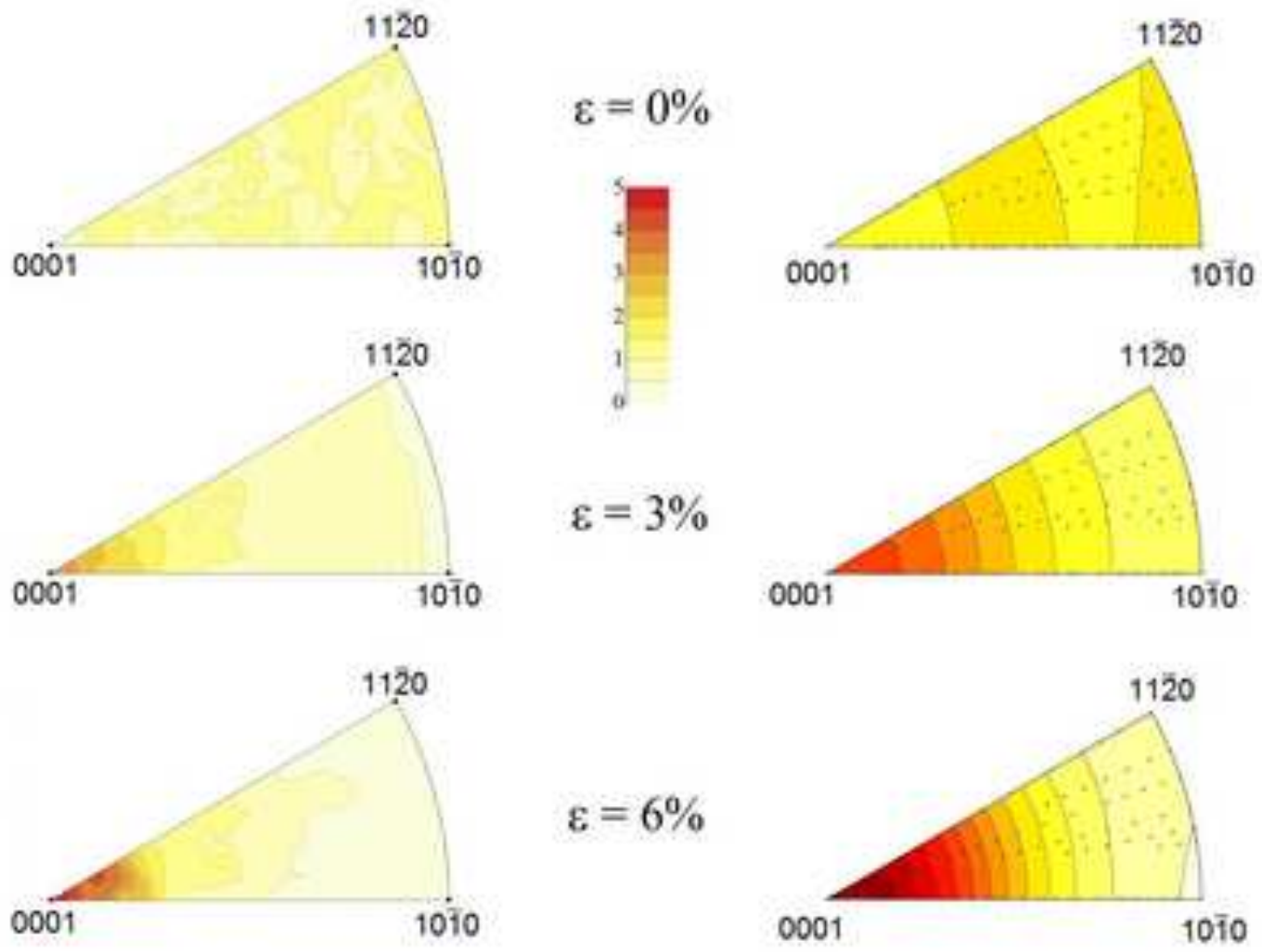

\section{Tension}

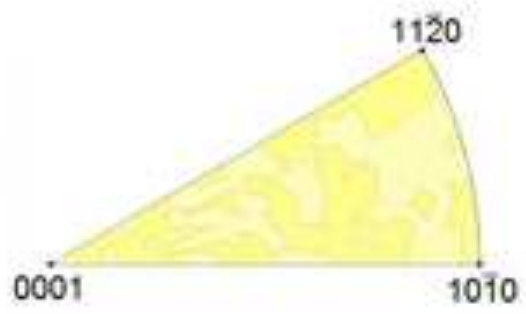

$$
\varepsilon=0 \%
$$
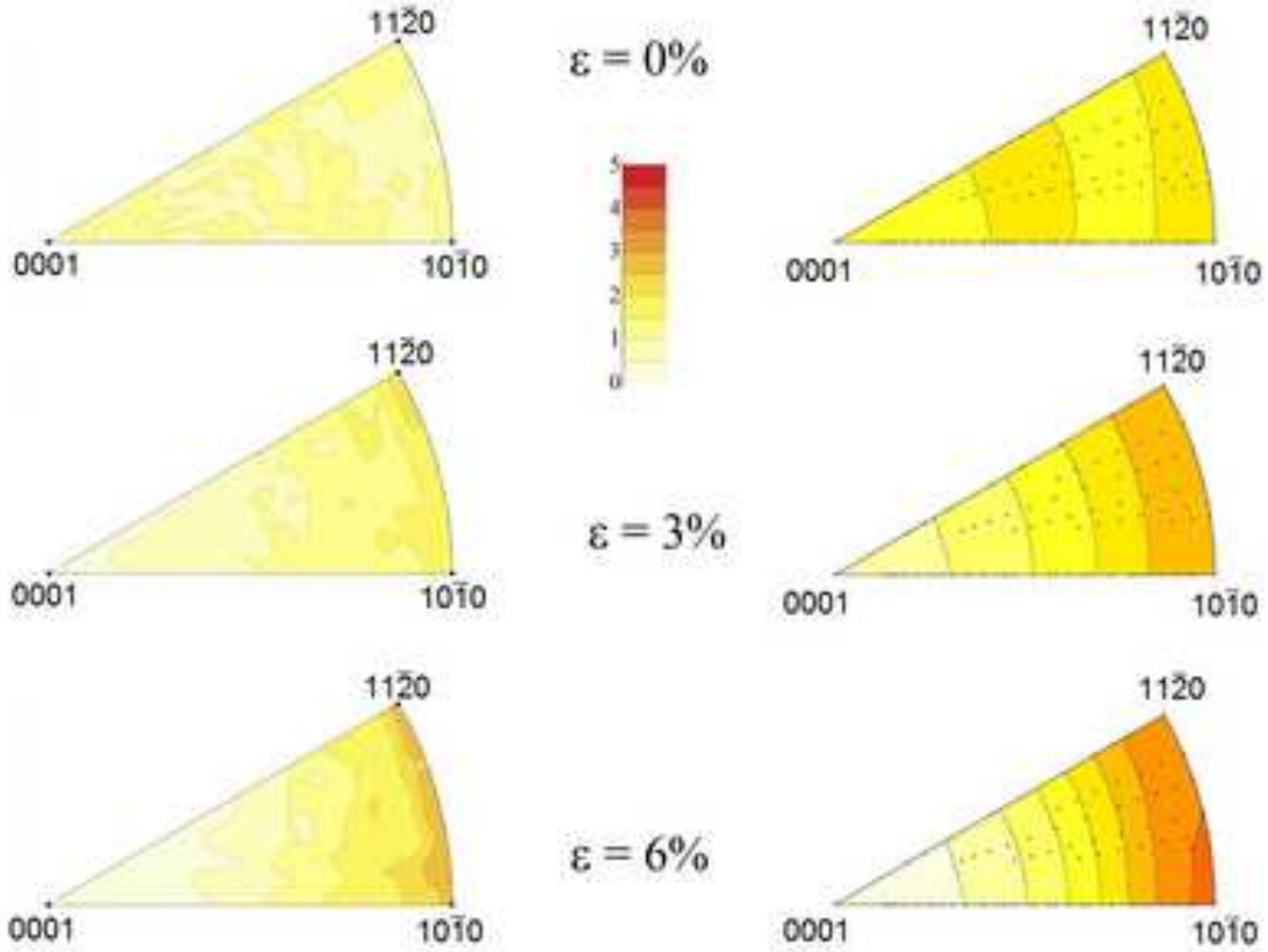


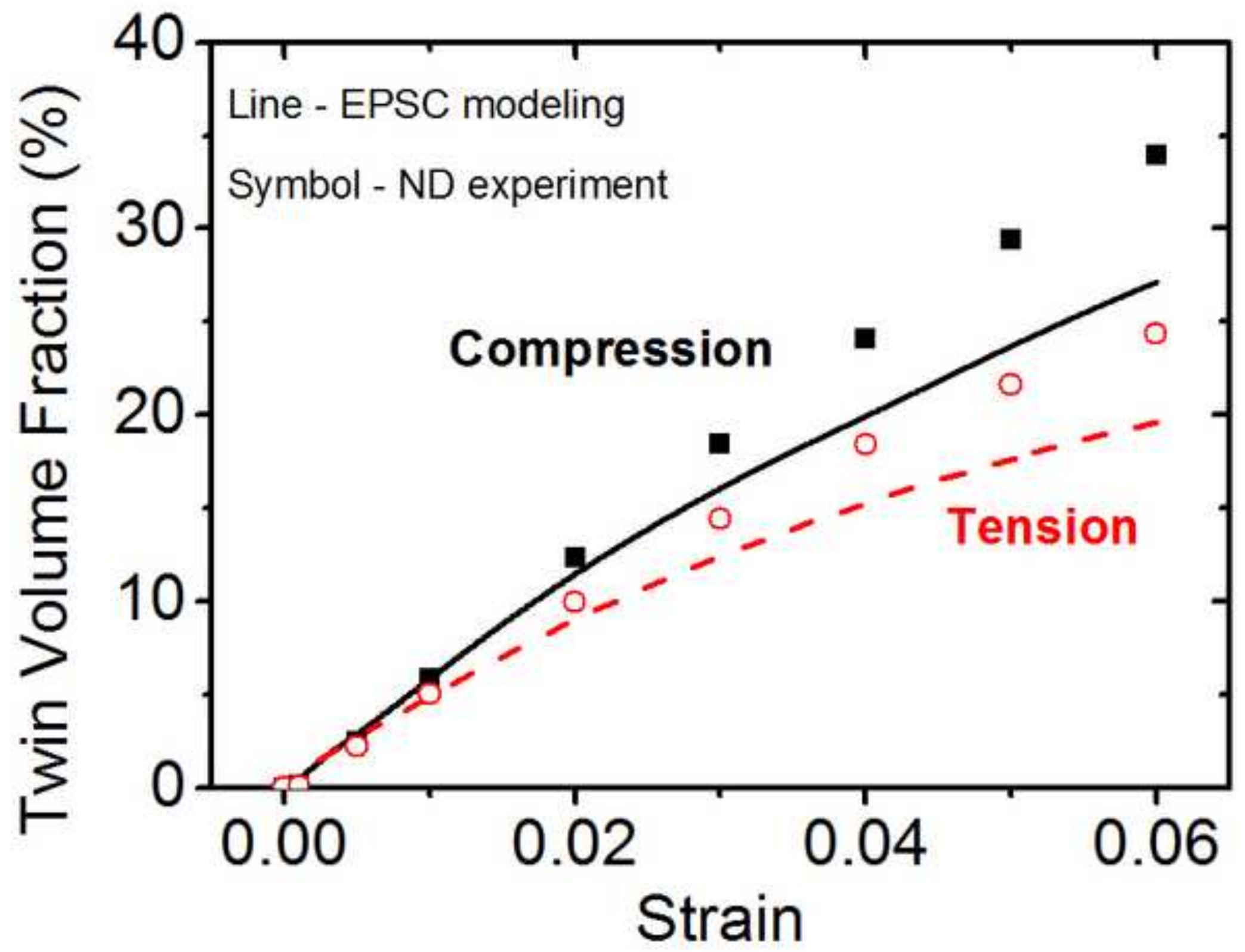




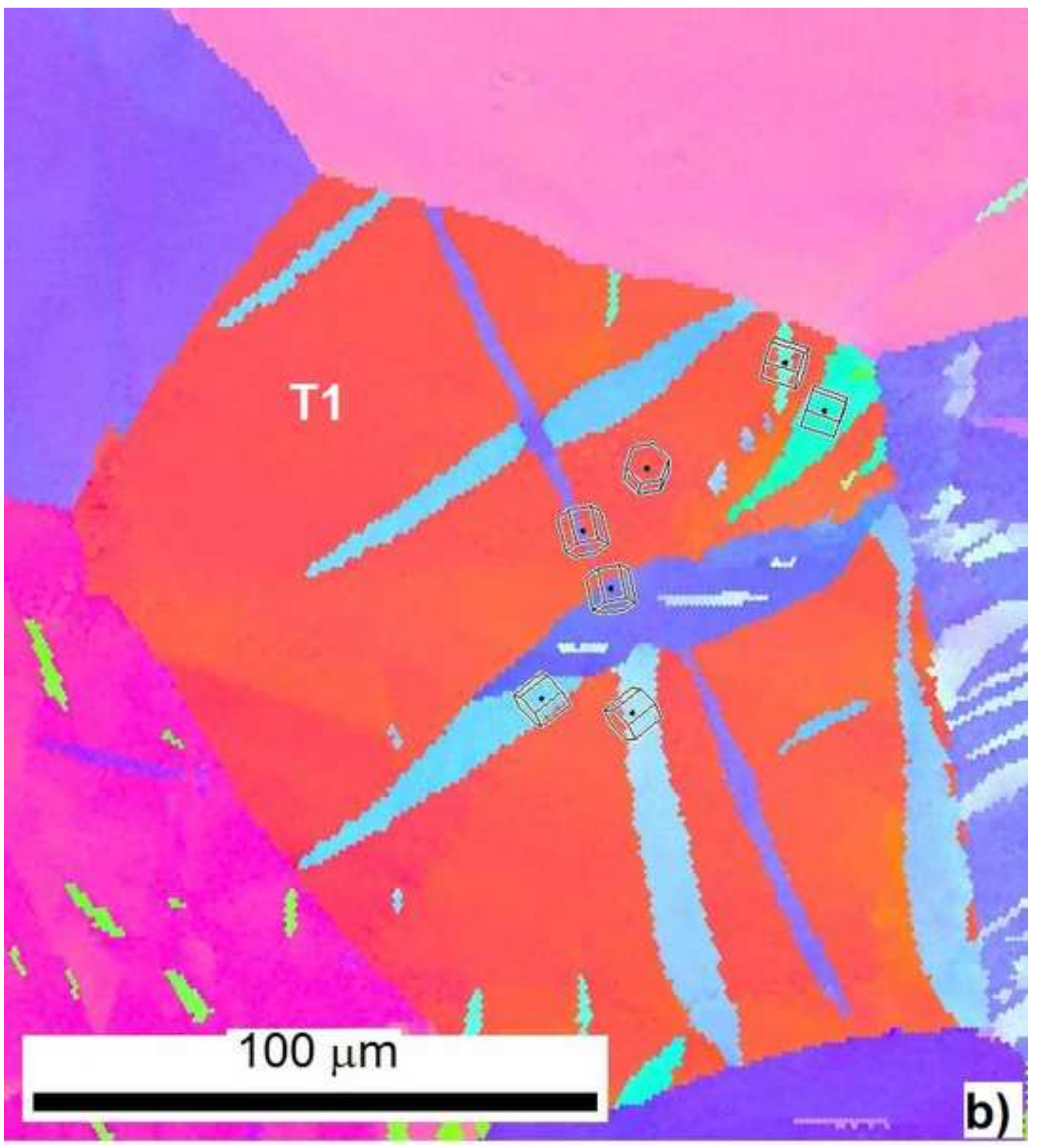




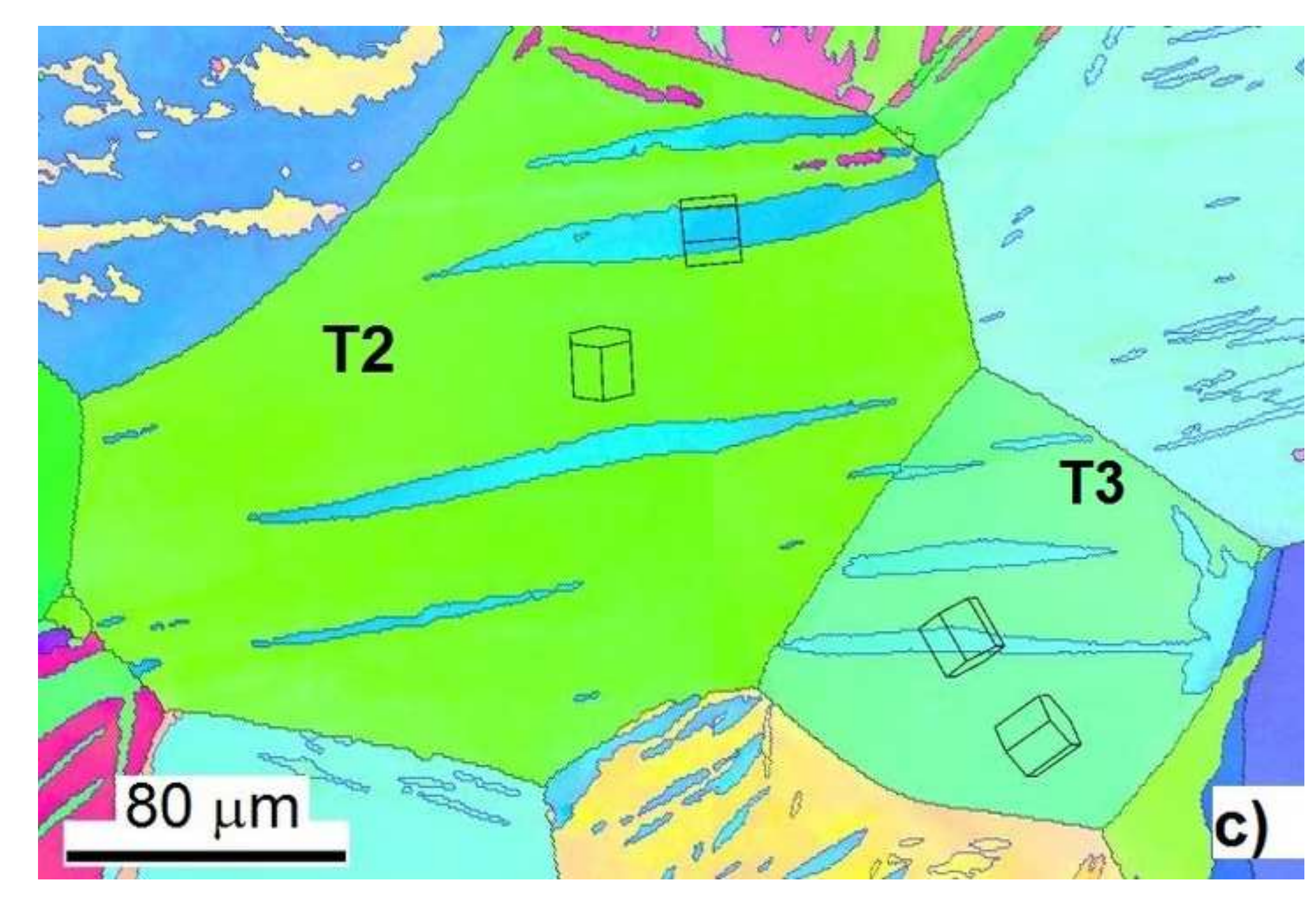

.
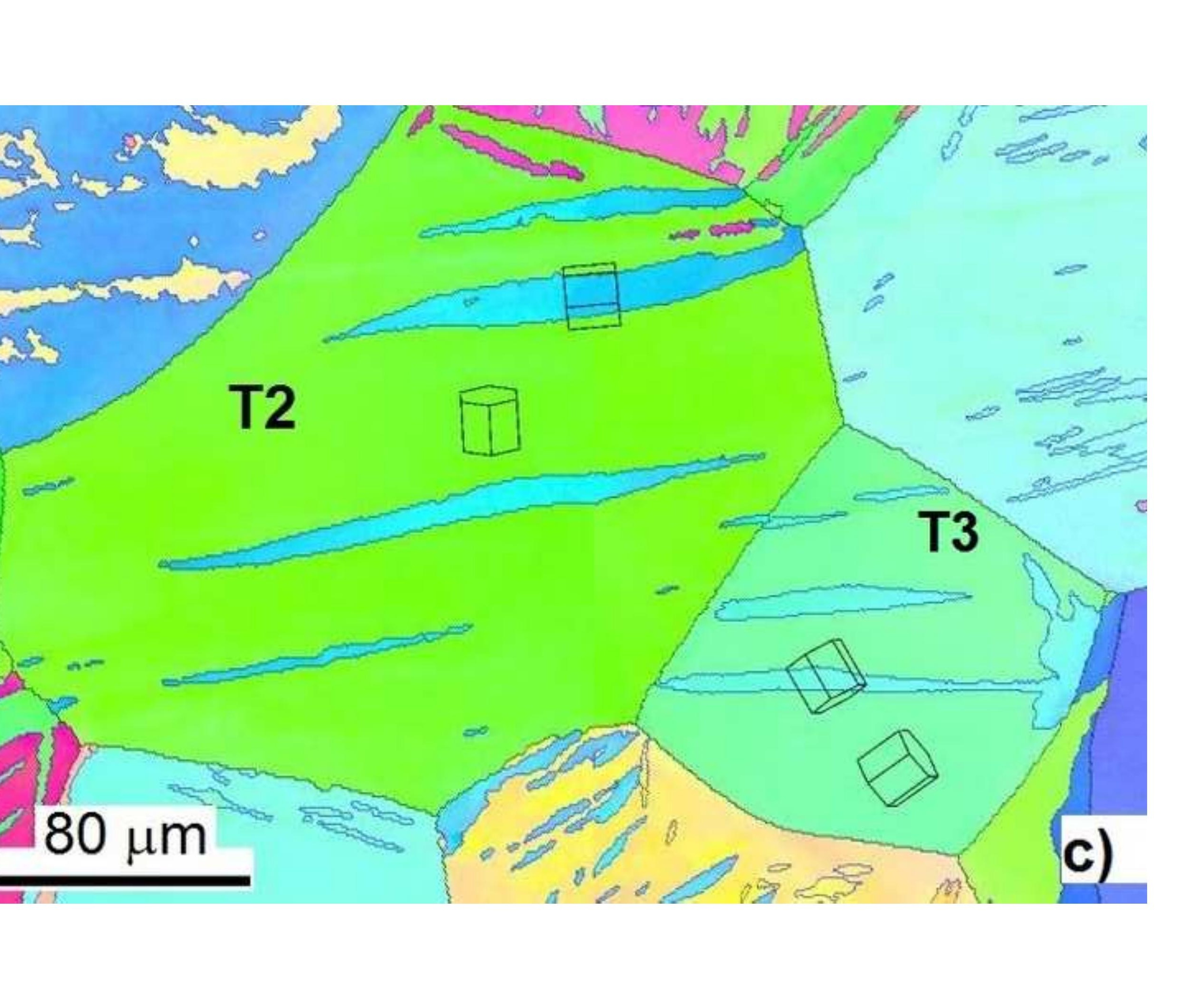


\section{Figure 16 orientation triangle}

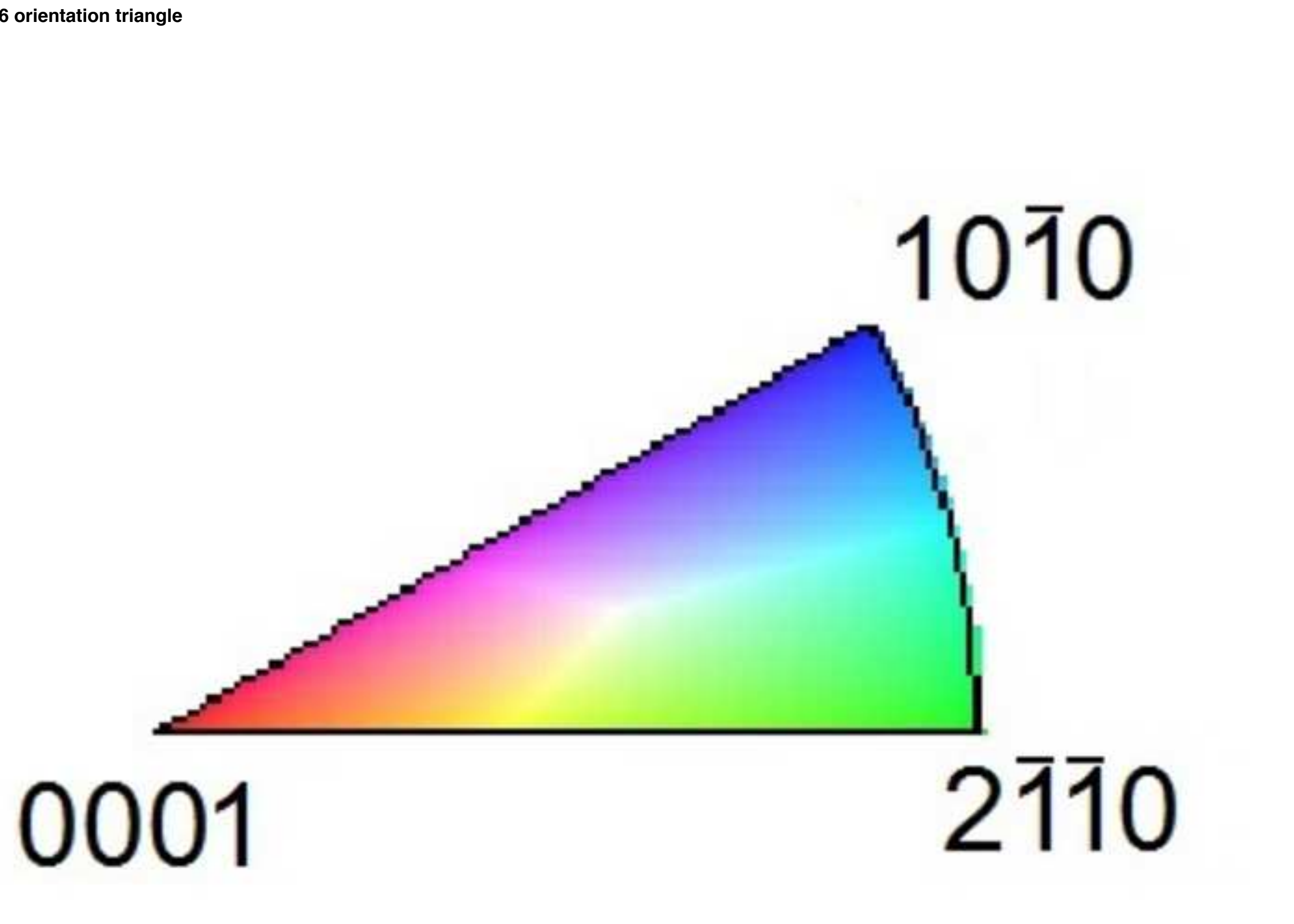

$21 \overline{10}$
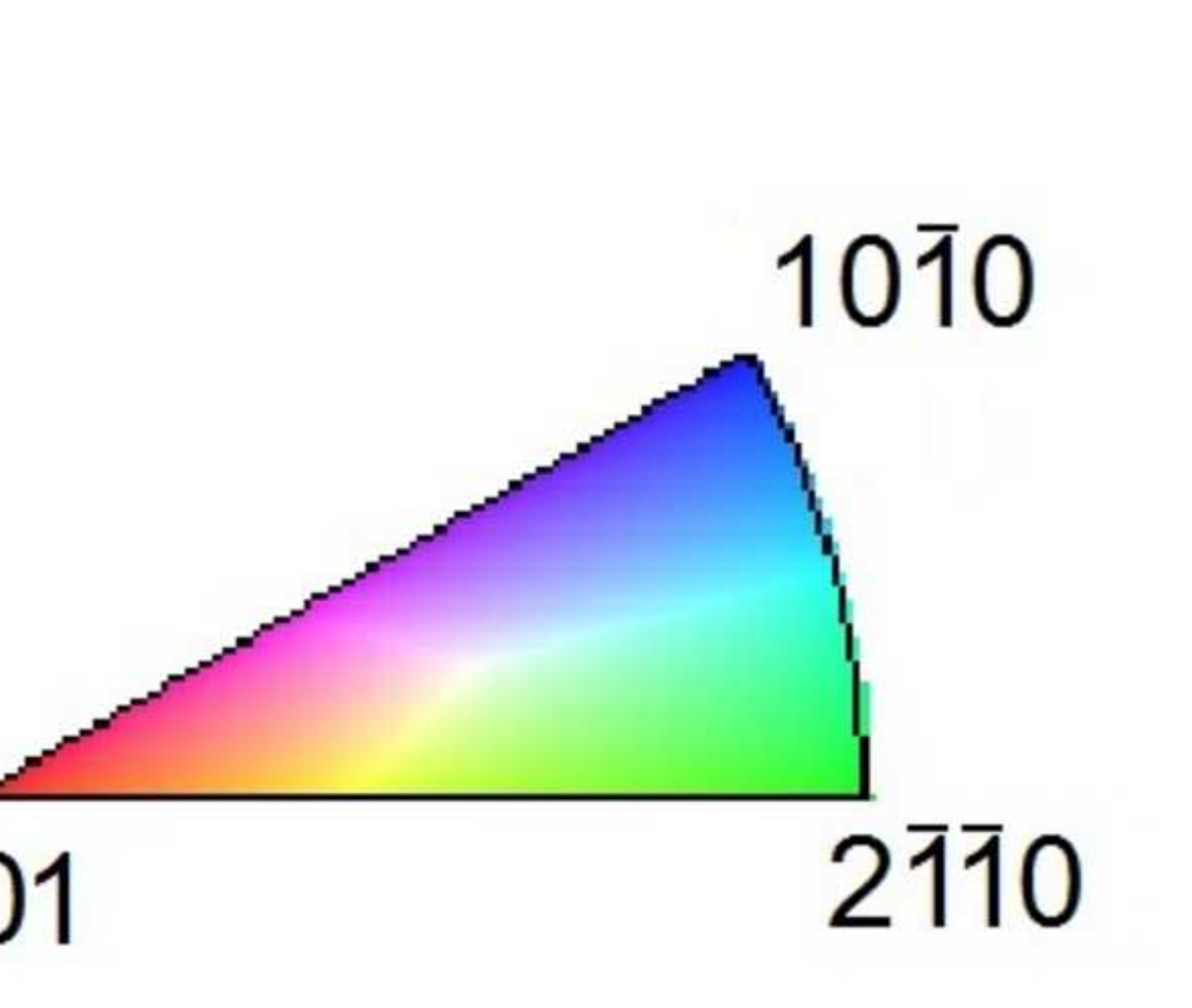

Figure 16 orientation triangle
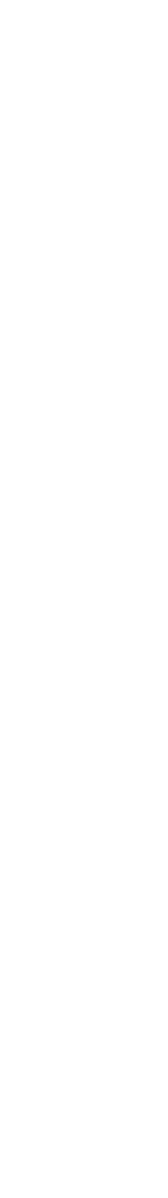

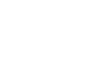




\section{Tension}

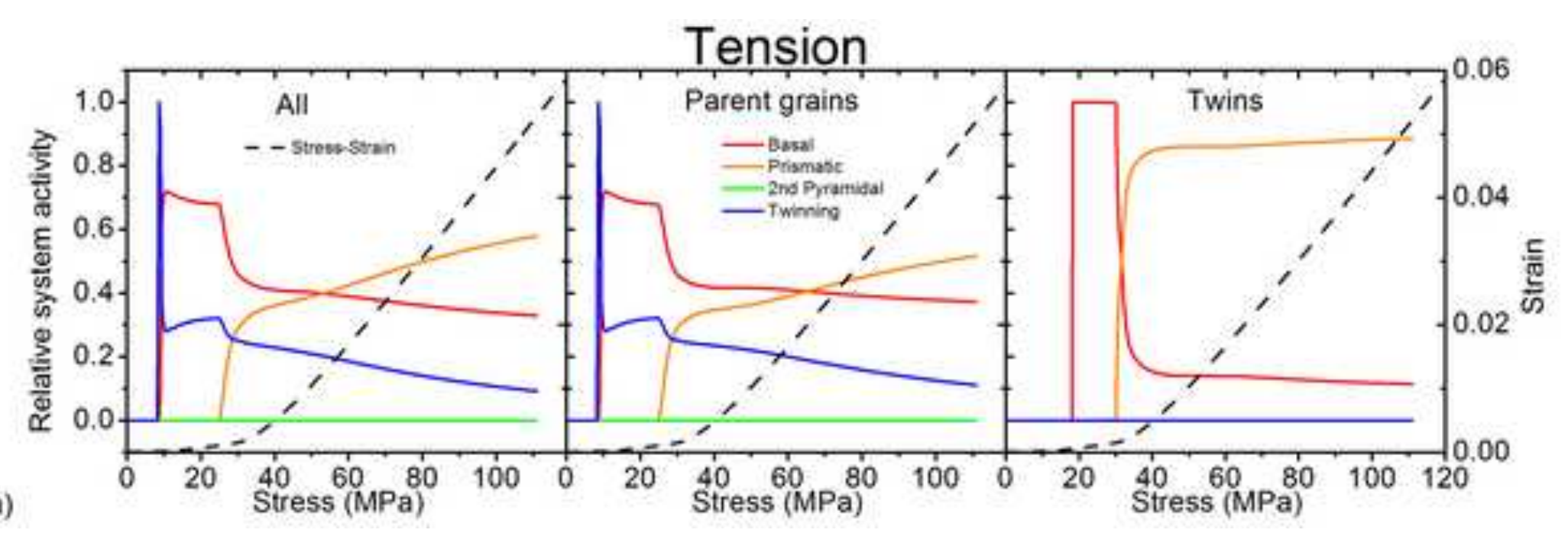

a)

\section{Figure 17a}

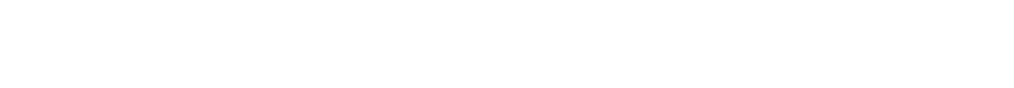


Compression

b)

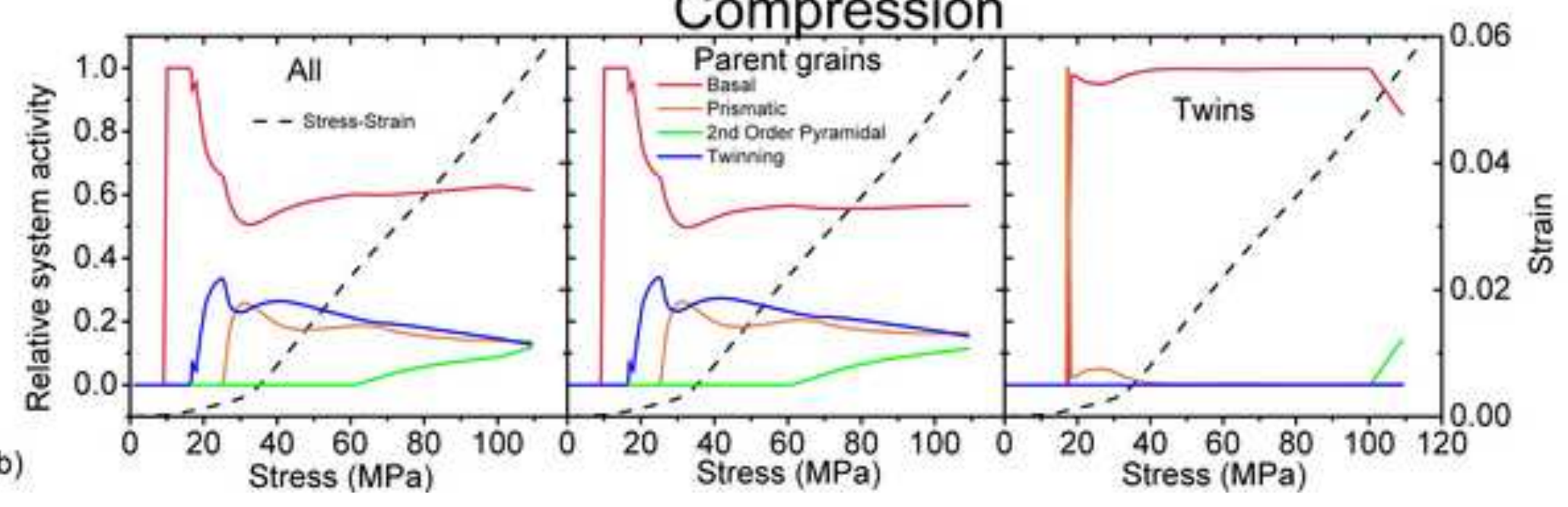



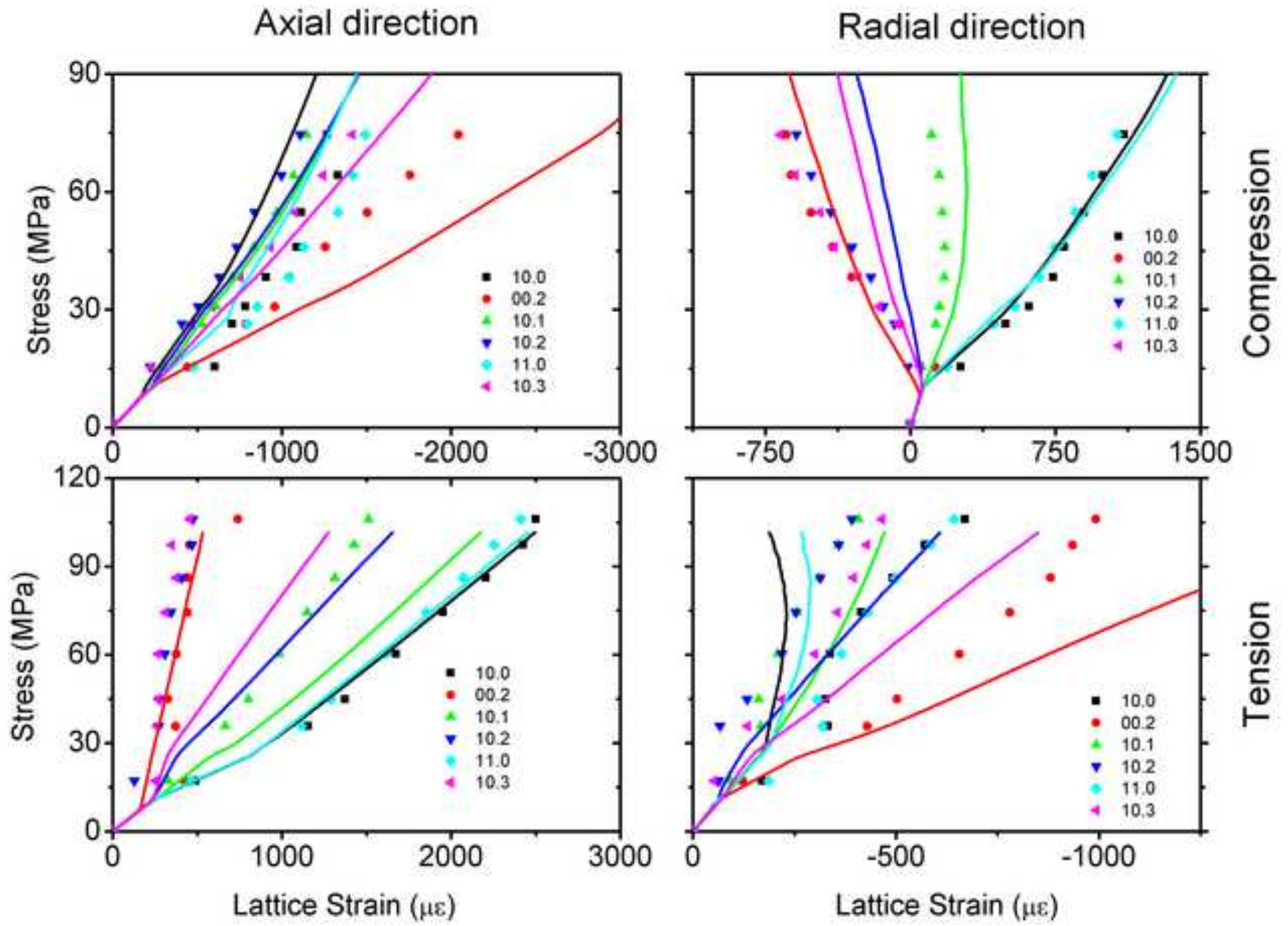

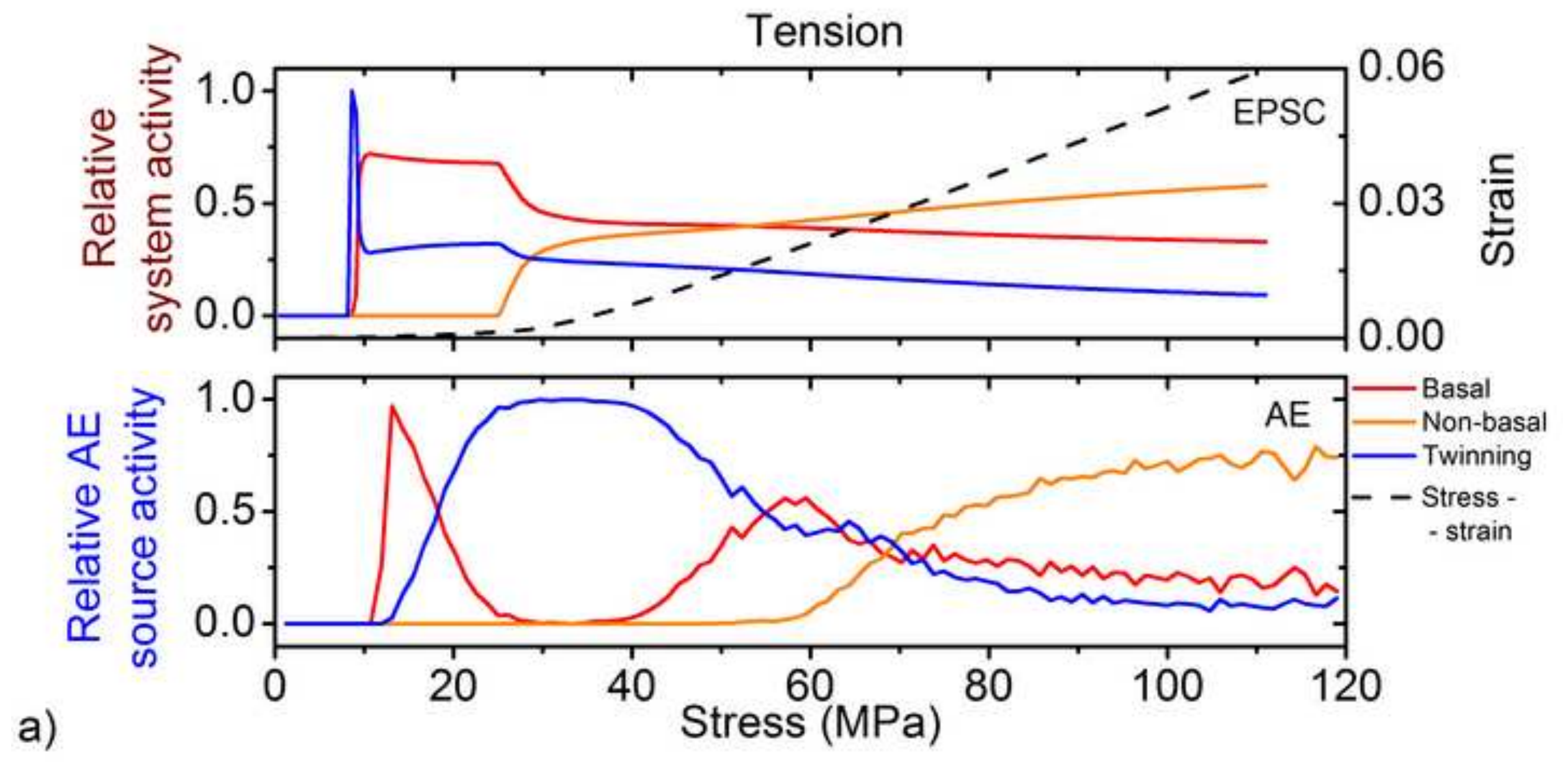

a) 
Compression
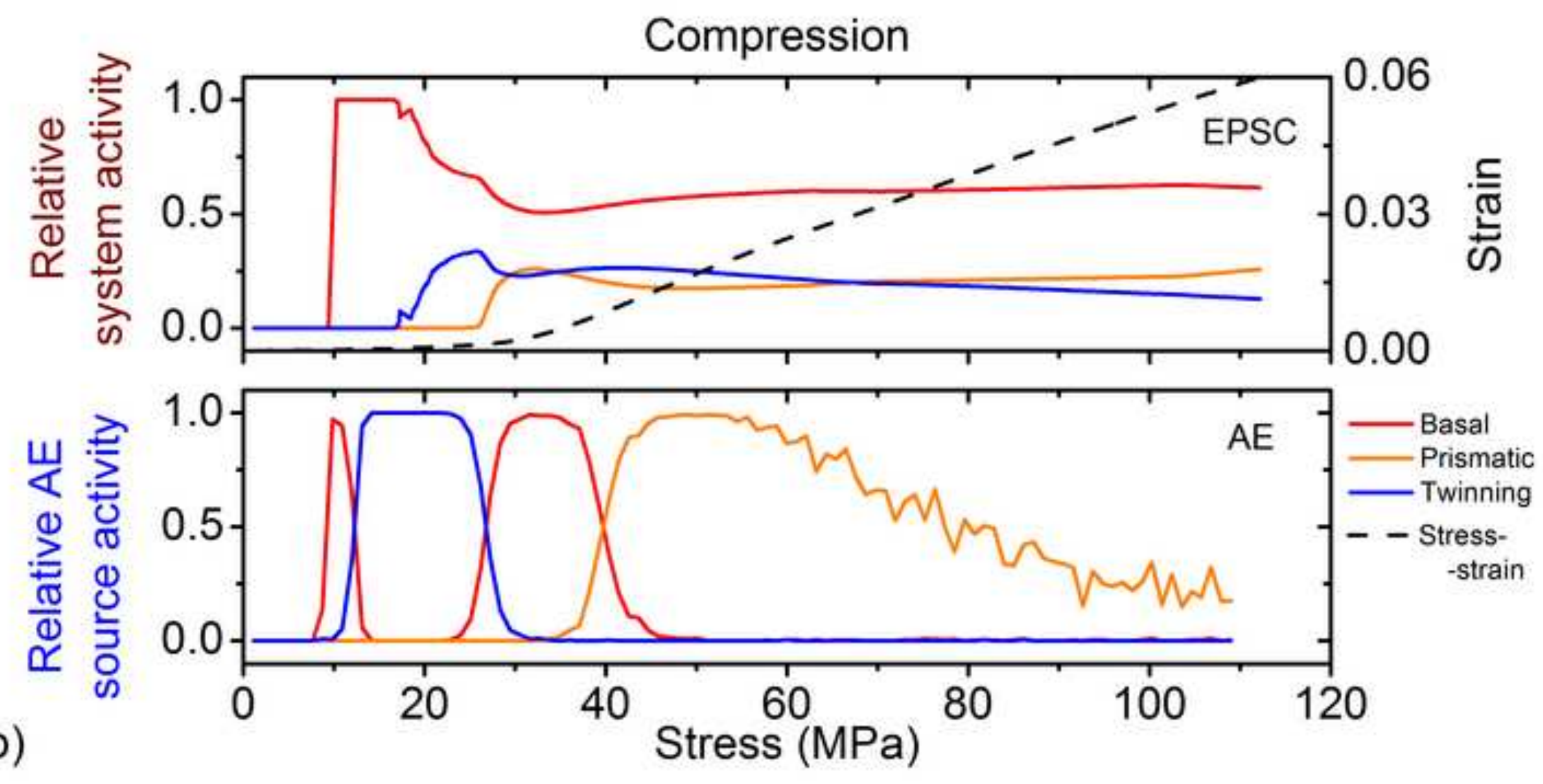

b) 